\title{
An Updated Review on the Genetics of Primary Open Angle Glaucoma
}

\author{
Khaled Abu-Amero ${ }^{1,2}$, Altaf A. Kondkar ${ }^{1}$ and Kakarla V. Chalam ${ }^{2, *}$ \\ Received: 27 October 2015; Accepted: 27 November 2015; Published: 4 December 2015 \\ Academic Editor: Emil Alexov \\ 1 Glaucoma Research Chair, Department of Ophthalmology, College of Medicine, King Saud University, \\ Riyadh 11424, Saudi Arabia; Khaled.Abu-Amero@jax.ufl.edu (K.A.-A.); akondkar@gmail.com (A.A.K.) \\ 2 Department of Ophthalmology, University of Florida College of Medicine, 580, W, 8th Street, Tower-2, \\ Jacksonville, FL 32209, USA \\ * Correspondence: kakarla.chalam@jax.ufl.edu; Tel.: +904-244-9361; Fax: +904-244-9391
}

\begin{abstract}
Epidemiological studies suggest that by 2020 the prevalence of primary open angle glaucoma (POAG) is estimated to increase to 76.0 million, and to 111.8 million by 2040 globally due to the population aging. The prevalence of POAG is the highest among those of African descent, followed by Asians, and the lowest in Europeans. POAG is a genetically complex trait with a substantial fraction exhibiting a significant heritability. Less than $10 \%$ of POAG cases in the general population are caused by specific gene mutations and the remaining cases are polygenic. Quantitative traits related to POAG pathogenesis such as intra-ocular pressure (IOP), vertical cup/disc ratio (VCDR), optic disc area, and central corneal thickness (CCT) are highly heritable, and likely to be influenced at least in part by genes and show substantial variation in human populations. Recent genome-wide association studies (GWAS) have identified several single nucleotide polymorphisms (SNPs) at different loci including CAV1/CAV2, TMCO1, CDKN2B-AS1, CDC7-TGFBR3, SIX1/SIX6, GAS7 and ATOH7 to be associated with POAG and its related quantitative traits (endophenotypes). The chapter provides a brief overview on the different GWAS and SNP association studies and their correlation with various clinical parameters important for POAG in the population worldwide, including the Middle East.
\end{abstract}

Keywords: epidemiology; genetics; GWAS; POAG; quantitative traits; SNP genotyping

\section{Introduction}

Glaucoma is a chronic and progressive group of optic neuropathies affecting more than 60 million people globally [1]. It is associated with death of retinal ganglion cells resulting in characteristic cupping or degeneration of the optic nerve head and loss of peripheral vision [2]. Primary open angle glaucoma (POAG) is one of the most common types of glaucoma which is clinically characterized by an open and normal anterior iridocorneal chamber angle [2]. POAG can either occur with increased intraocular pressure (IOP) or normal IOP, the latter being referred to as normal-tension glaucoma (NTG).

Although there are many postulated mechanisms of retinal ganglion cell damage, the exact etiology of POAG still remains obscure. The well-recognized risk factors associated with POAG include elevated IOP, age, family history, gender, ethnicity, central corneal thickness, and myopia. A recent large prospective study indicated that POAG with early paracentral visual field loss displays distinct as well common risk factor profiles as compared to those with peripheral vision loss [3]. Raised IOP is the most important and the only modifiable risk factor in the development and progression of POAG. Several large population-based studies in the past have confirmed that the reduction of IOP reduces the progression of glaucoma in patients with or without elevated IOP [4-8]. 
Similarly, findings of the meta-analysis from the Eye Diseases Prevalence Research Group have shown that the occurrence of glaucoma increases with increasing age among all ethnicities (Europeans, Blacks, and Hispanics) [7,9]. Age was also reported to be associated with POAG in patients with ocular hypertension in two large population-based studies [5,8]. Family history is another important risk factor in the development of glaucoma [7,10-12]. A positive family history of POAG significantly increases the odds (varying from five to 10 times) for the development of POAG [13]. In the Melbourne [14] and Rotterdam studies [11], males showed a trend towards increased risk of POAG which was absent in the Barbados Eye Study [7] and the Beaver Dam Eye Study [10]. Similarly, the Eye Disease Prevalence Research Group [9] reported no gender-related association of glaucoma among the European, African American, and Hispanic subjects. However, a recent systematic review of 3497 POAG cases out of 146,882 participants with gender-specific data showed that the age-adjusted prevalence is higher in men compared to women, and that this finding remains consistent across all ethnic groups provides very strong evidence for the association of POAG with gender [15]. Several studies have shown POAG to be more prevalent with rapid and severe disease progression in people of African-Caribbean as compared to European descent, Hispanics, and Asians $[9,16]$. Central corneal thickness (CCT) has also been reported to be associated with POAG, particularly in the ocular hypertension patients $[17,18]$. Although the precise mechanism(s) are still unclear, this may be in part due to the effect of corneal thickness on IOP measurement, and increased susceptibility to optic nerve damage $[19,20]$. In addition, studies have shown that individuals with thicker corneas are less responsive to topical ocular hypotensive medications [21]. Myopia is also considered to be an important risk factor for POAG as it can increase susceptibility of myopic nerves to glaucomatous damage [22]. Moderate-to-high levels of myopia conferred two- to three-fold increased risk in the Australian [23], US Caucasian [24], and the Chinese populations [25]. Other predisposing factors for POAG include adult-onset diabetes and hypertension. Although there are conflicting reports regarding the risk of POAG in individuals with diabetes [26,27], a recent systematic review and meta-analysis of 13 studies, which included six population-based cohorts and seven case-control studies, showed increased risk of POAG (relative risk of 1.4 and 1.49, respectively) in individuals with diabetes [28]. Multiple epidemiological studies have also reported a role of hypertension as a risk factor for POAG [23,29]. Treatment of hypertensive patients with beta-blockers results in nocturnal hypotension and is a potential risk factor for glaucomatous optic neuropathy [30]. The mechanism(s) by which hypertension induces optic nerve damage are still unclear.

POAG is a genetically complex trait with a substantial fraction exhibiting a significant heritability. Genetic linkage studies of large affected families have so far identified at least 20 chromosomal loci (GLC1A-P) that are linked to POAG. The causative genes that are capable of causing POAG with minimal influence from other gene(s) or the environment and that have been consistently implicated so far include myocilin (MYOC), optineurin (OPTN), WD repeat domain 36 (WDR36), ankyrin repeat and SOCS-box containing 10 (ASB10), Cytochrome P450 family 1, subtype B, polypeptide 1 (CYP1B1), and neurotrophin 4 (NTF4) as reviewed elsewhere [31,32]. Twin studies and family-based studies have discovered a number of genes. However, these disease-causing genes account for $<10 \%$ of POAG cases in the general population. It is therefore likely that the hereditary aspect of many of the remaining cases of POAG is due to the combined effects of several genes (polygenic) and that gene-environment interactions are important. Quantitative endophenotype traits related to POAG pathogenesis such as IOP, vertical cup-to-disc ratio (VCDR), and CCT $[10,33,34]$ are highly heritable, likely to be influenced at least in part by genes, and are highly polymorphic. Recent advances in genomic technologies and genome-wide association studies (GWAS) have greatly accelerated the discovery and understanding of genes and genomic regions associated with POAG and influencing the quantitative endophenotype traits related to POAG pathogenesis, which will be the main focus of this chapter. 


\section{Epidemiology of POAG}

Recent epidemiological studies suggest that, in 2013, almost 64.3 million people (aged between 40 and 80 years) were affected by glaucoma globally, and this number is expected to increase to 76.0 million by 2020 and to 111.8 million by 2040 due to the population aging [35]. POAG accounts for a major three-quarters (74\%) of all glaucoma cases [1]. Another recent meta-analysis estimated the global number of POAG cases in 2015 at 57.5 million, rising to 65.5 million by 2020 [15]. Almost half $(47 \%)$ of these will those of Asian descent, while a quarter (24\%) will be European [1]. The risk and subtypes of glaucoma are known to vary among races and countries [36]. A meta-analysis conducted by the Eye Disease Prevalence Research Group showed that, in the United States, African Americans have a higher POAG prevalence than Caucasians. The prevalence of POAG in individuals $\geqslant 40$ years old was observed to be $1.86 \%$, including 1.57 million Caucasian and 398,000 African American subjects. In 2020, this number is estimated to rise up to 3.36 million due to the population aging [9]. In all the age groups, there was an increased prevalence of glaucoma in individuals of African descent compared with European-derived individuals [37]. Similarly, a recent meta-analysis of 81 studies including 37 countries, 216,214 participants, and 5266 POAG cases reported that the Black populations had the highest POAG prevalence of 5.2\% (95\% credible interval (CrI) 3.7\%, 7.2\%) at 60 years, rising to $12.2 \%$ ( $95 \% \mathrm{CrI} 8.9 \%$ to $16.6 \%$ ) at 80 years. The increase in POAG prevalence per decade of age was found to be highest among the Hispanics $(2.31,95 \%$ CrI 2.12, 2.52) and Caucasian populations $(1.99,95 \%$ CrI 1.86, 2.12), and lowest in East and South Asians $(1.48,95 \%$ CrI 1.39, 1.57; 1.56, 95\% CrI 1.31, 1.88, respectively). In addition, men were more likely to have POAG than women $(1.30,95 \%$ CrI 1.22, 1.41). It is clearly evident that individuals of African descent are associated with increased risk (estimated incidence is two to five times higher) of developing glaucoma compared with individuals of European descent. The reasons for this increased risk of glaucoma among individuals of African descent are still not clear. The Barbados Eye Study reported a prevalence of $7 \%$ in Africans, suggesting an influence of ancestral factors [7]. Several other factors that may also be influential could be physiological or anatomical differences in the optic disc or corneas, environmental factors, social differences or genetics [26].

\section{Genotype-Phenotype Association in POAG}

Association studies using the candidate-gene approach and GWASs have been particularly useful tools in identifying genetic factors, each of which may have a relatively small effect but contributes to a large number of cases. Unlike the candidate-gene approach, GWAS is an unbiased (without bias to known protein functionality gene) genome-wide approach that compares the genotypic profile of single nucleotide polymorphisms (SNPs) throughout the genome in cases (affected) and controls (unaffected), thus identifying genomic region(s) associated with a disease or trait of interest. The large population sample required in GWASs to achieve a genome-wide statistical significance ( $p$-value of less than $5 \times 10^{-8}$ ) has been greatly facilitated by the formation of the International Consortia. However, since GWAS can rarely identify functional or causal variant(s), further in-depth genotyping and functional testing in addition to replication studies in independent cohorts of different population groups are considered a standard requirement to conclusively validate genes or genomic regions identified from GWAS. Using this powerful approach (GWAS), recent genetic studies have identified genes or genetic variants with modest effect to be associated with POAG and related quantitative traits (Table 1). These studies have provided better insights into the genetic basis of POAG and improved our understanding of the underlying pathophysiology of the disease. 
Table 1. Genes and polymorphisms identified in POAG using genome-wide and candidate-gene approaches in the Middle East and other populations.

\begin{tabular}{|c|c|c|c|c|c|c|c|}
\hline Studies & Gene/Chromosome & SNP ID & Population * & Study Type & $\begin{array}{c}\text { Study Size } \\
\text { (POAG/Controls) * }\end{array}$ & OR/Beta, $p$ Value & Any Clinical Association * \\
\hline \multicolumn{8}{|c|}{ GWAS Studies } \\
\hline \multirow{6}{*}{ Nakano et al., 2009 [38] } & PLXDC2 (10p12.31) & rs7081455 & \multirow{6}{*}{$\begin{array}{l}\text { D: Japan } \\
\text { R: Japan }\end{array}$} & \multirow{6}{*}{ GWAS } & \multirow{6}{*}{$\begin{array}{l}\text { D: } 1519 \\
\text { R: } 857\end{array}$} & $\mathrm{OR}=1.49, p=1 \times 10^{-5}$ & - \\
\hline & TMTC2 (12q21.31) & rs7961953 & & & & $\mathrm{OR}=1.37, p=7 \times 10^{-5}$ & - \\
\hline & & rs547984 & & & & $\mathrm{OR}=1.34, p=6 \times 10^{-5}$ & \\
\hline & ZP4 (1a43) & rs540782 & & & & $\mathrm{OR}=1.34, p=6 \times 10^{-5}$ & _- \\
\hline & & rs693421 & & & & $\mathrm{OR}=1.35, p=4 \times 10^{-5}$ & \\
\hline & & rs2499601 & & & & $\mathrm{OR}=1.33, p=9 \times 10^{-5}$ & \\
\hline \multirow{2}{*}{ Meguro et al., 2010 [39] } & SRBD1 (2p21) & rs3213787 & \multirow[t]{2}{*}{ Japanese } & GWAS & \multirow{2}{*}{$\begin{array}{l}\text { D: } 305 \\
\text { R: } 355 \\
\quad-\end{array}$} & $\mathrm{OR}=2.80, p=2.5 \times 10^{-9}$ & Associated with NPG \\
\hline & ELOVL5 (6p12.1) & rs735860 & & - & & $\mathrm{OR}=1.69, p=4.1 \times 10^{-6}$ & Associated with NPG \\
\hline \multirow{2}{*}{ Thorleifsson et al., 2010 [40] } & \multirow{2}{*}{ CAV1/CAV2 (7q31.1) } & rs4236601 & \multirow{2}{*}{$\begin{array}{l}\text { D: Iceland } \\
\text { R1: SW, UK, AU } \\
\text { R2: China }\end{array}$} & \multirow{2}{*}{ GWAS } & \multirow{2}{*}{$\begin{array}{l}\text { D: } 36,140 \\
\text { R1: } 4239 \\
\text { R2: } 879\end{array}$} & $\mathrm{OR}=1.36, p=5 \times 10^{-10}$ & \multirow{2}{*}{$\begin{array}{c}\text { Nominal association was observed } \\
\text { for increased IOP }(p=0.034) \\
-\end{array}$} \\
\hline & & rs1052990 & & & & $\mathrm{OR}=1.32, p=1.1 \times 10^{-9}$ & \\
\hline \multirow{2}{*}{ Burdon et al., 2011 [41] } & CDKN2B-AS (9p21.3) & rs4977756 & \multirow{2}{*}{$\mathrm{AU}, \mathrm{NZ}$} & \multirow{2}{*}{ GWAS } & \multirow{2}{*}{$\begin{array}{l}\text { D: } 590 / 3956 \\
\text { R: } 4148\end{array}$} & $\mathrm{OR}=1.50, p=4.7 \times 10^{-9}$ & - \\
\hline & TMCO1 (1q24) & rs4656461 & & & & $\mathrm{OR}=1.68, p=6.1 \times 10^{-10}$ & - \\
\hline \multirow{3}{*}{ Wiggs et al., 2012 [42] } & CDKN2B-AS (9p21) & rs2157719 & \multirow{3}{*}{ US Caucasian } & \multirow{3}{*}{ GWAS } & \multirow{3}{*}{ D: $3146 / 3487$} & $\mathrm{OR}=0.69, p=1.86 \times 10^{-18}$ & Also associated with NPG. \\
\hline & SIX1/SIX6 (14q23) & rs10483727 & & & & $\mathrm{OR}=1.32, p=3.87 \times 10^{-11}$ & $\begin{aligned} O R=0.58, p & =1.17 \times 10^{-12} \\
& -\end{aligned}$ \\
\hline & $8 q 22$ & rs284489 & & & & $\mathrm{OR}=0.62, p=8.88 \times 10^{-10}$ & Associated with NPG \\
\hline \multirow{3}{*}{ Osman et al., 2012 [43] } & CDKN2B-AS (9p21) & rs1063192 & \multirow{3}{*}{ Japanese } & \multirow{3}{*}{ GWAS } & \multirow{3}{*}{$\begin{array}{l}\text { D: } 7993 \\
\text { R: } 9014\end{array}$} & $\mathrm{OR}=0.75, p=5.2 \times 10^{-11}$ & - \\
\hline & SIX1/SIX6 (14q23) & rs10483727 & & & & $\mathrm{OR}=0.79, p=9.49 \times 10^{-8}$ & - \\
\hline & NCKAP5 (2q21.2) & rs7588567 & & & & $\mathrm{OR}=0.85, p=3.89 \times 10^{-7}$ & - \\
\hline \multirow[b]{2}{*}{ Nakano et al., 2012 [44] } & \multirow[b]{2}{*}{ CDKN2B-AS (9p21.3) } & rs7865618 & \multirow[b]{2}{*}{ Japanese } & \multirow[b]{2}{*}{ GWAS } & D: $833 / 686$ & $\mathrm{OR}=1.78, p=9.0 \times 10^{-11}$ & \multirow{2}{*}{$\begin{array}{l}\text { Strongly associated with } \\
\text { POAG and POAG/NPG } \\
\text { but not with HPG }\end{array}$} \\
\hline & & rs523096 & & & R: $411 / 289$ & $\mathrm{OR}=1.76, p=1.6 \times 10^{-10}$ & \\
\hline Takamoto et al., 2012 [45] & CDKN2B (9p21) & rs523096 & Japanese & GWAS & $\begin{array}{l}\text { D: } 286 / 557 \\
\text { R: } 183 / 514\end{array}$ & $\mathrm{OR}=2.13, p=4.96 \times 10^{-11}$ & Associated with NTG \\
\hline & $A B C A 1(9 q 31.1)$ & rs2487032 & Asian Southern & GWAS & D: $1007 / 1009$ & $\begin{aligned} \mathrm{OR} & =0.69, p=1.66 \times 10^{-8} \\
\mathrm{OR}_{\mathrm{R}} & =0.73, p_{\mathrm{R}}=2.79 \times 10^{-9}\end{aligned}$ & - \\
\hline Chen et al., 2014 [46] & PMM2 (16p13.2) & rs3785176 & Chinese & & R: $1899 / 4965$ & $\begin{aligned} \mathrm{OR} & =1.42, p=3.18 \times 10^{-6} \\
\mathrm{OR}_{\mathrm{R}} & =1.30, p_{\mathrm{R}}=5.77 \times 10^{-10}\end{aligned}$ & - \\
\hline
\end{tabular}


Table 1. Cont

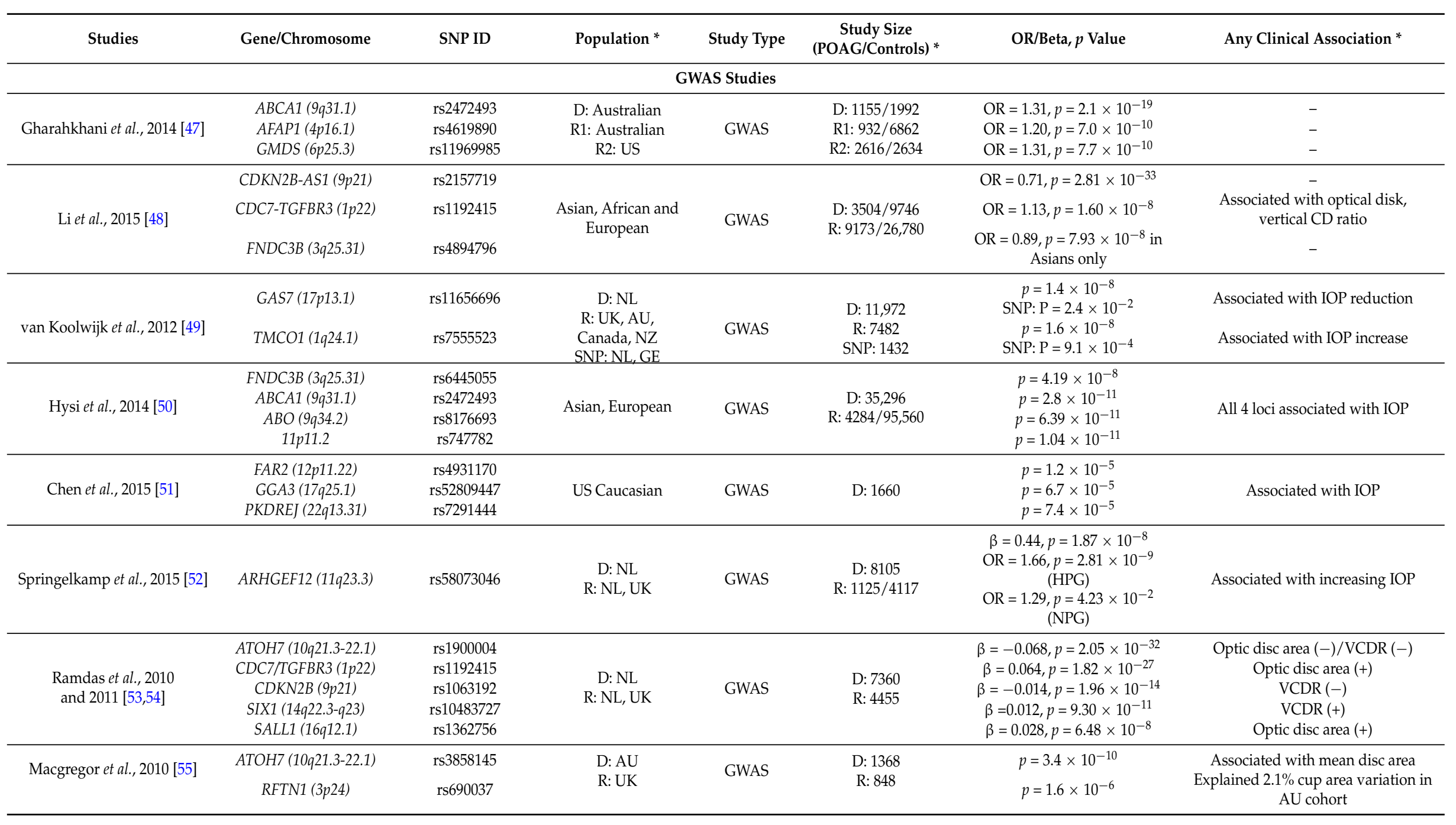


Table 1. Cont

\begin{tabular}{|c|c|c|c|c|c|c|c|}
\hline Studies & Gene/Chromosome & SNP ID & Population * & Study Type & $\begin{array}{c}\text { Study Size } \\
\text { (POAG/Controls) * }\end{array}$ & OR/Beta, $p$ Value & Any Clinical Association * \\
\hline \multicolumn{8}{|c|}{ GWAS Studies } \\
\hline Khor et al., 2011 [56] & $\begin{array}{c}\text { CARD10 (22q13.1) } \\
\text { ATOH7 (10q21.3-22.1) } \\
\text { CDC7/TGFBR3 (1p22) }\end{array}$ & $\begin{array}{l}\text { rs9607469 } \\
\text { rs7916697 } \\
\text { rs1192415 }\end{array}$ & $\begin{array}{l}\text { D: Asian } \\
\text { R: NL }\end{array}$ & GWAS & $\begin{array}{l}\text { D: } 4445 \\
\text { R: } 9326\end{array}$ & $\begin{array}{l}p=2.73 \times 10^{-12} \\
p=2.00 \times 10^{-15} \\
p=7.57 \times 10^{-17}\end{array}$ & $\begin{array}{l}\text { Associated with optic disc area } \\
\text { Associated with optic } \\
\text { disc area in Asians }\end{array}$ \\
\hline Iglesias et al., 2014 [[57] ] & SIX6 (14q23) & $\begin{array}{l}\text { rs33912345 } \\
\text { (His141Asn) } \\
\text { rs146737847 } \\
\text { (Glu29Lys) }\end{array}$ & $\begin{array}{l}\text { D: NL, UK } \\
\text { R: NL, UK }\end{array}$ & GWAS & $\begin{array}{l}\text { D: } 292 / 1208 \\
\text { R: } 11,473\end{array}$ & $\begin{array}{l}p=7.74 \times 10^{-7} \\
p=5.0 \times 10^{-3}\end{array}$ & $\begin{array}{c}\text { Associated with VCDR and POAG } \\
\text { Associated with VCDR }\end{array}$ \\
\hline Vitart et al., 2010 [58] & $\begin{array}{c}\text { COL5A1 9q34.2 } \\
\text { ZNF469 16q24.2 } \\
\text { AKAP13 15q24-25 } \\
\text { AVGR8 13q12.11 }\end{array}$ & $\begin{array}{c}\text { rs1536482 } \\
\text { rs12447690 } \\
\text { rs6496932 } \\
\text { rs1034200 }\end{array}$ & Croatia, Scotland & GWAS & $\begin{array}{l}\text { D: } 7711 \\
\text { R: } 2681\end{array}$ & $\begin{array}{l}\beta=0.22, p=7.1 \times 10^{-8} \\
\beta=0.23, p=4.4 \times 10^{-9} \\
\beta=0.13, p=1.4 \times 10^{-8} \\
\beta=0.14, p=3.5 \times 10^{-9}\end{array}$ & Associated with CCT \\
\hline Vithana et al., 2011 [59] & $\begin{array}{c}\text { ZNF469 (16q24) } \\
\text { COL5A1/RXRA } \\
(9 q 34.2-q 34.3) \\
\text { COL8A2 (1p34.2) }\end{array}$ & $\begin{array}{l}\text { rs12447690 } \\
\text { rs9938149 } \\
\text { rs1536478 } \\
\text { rs7044529 } \\
\text { rs96067 }\end{array}$ & $\begin{array}{l}\text { D1: SG-Malay D2: } \\
\text { SG-Chinese }\end{array}$ & GWAS & $\begin{array}{l}\text { D1: } 3280 \\
\text { D2: } 3400\end{array}$ & $\begin{array}{c}\beta=-5.068, p=1.92 \times 10^{-14} \\
\beta=-6.248, p=1.63 \times 10^{-16} \\
\beta=-4.63, p=3.05 \times 10^{-9} \\
\beta=2.7, p=1.2 \times 10^{-4} \\
\beta=-4.799, p=5.40 \times 10^{-13}\end{array}$ & Associated with CCT \\
\hline Ulmer et al., 2012 [60] & $\begin{array}{c}\text { ZNF469 (16q24) } \\
\text { NTM (11q25) }\end{array}$ & $\begin{array}{l}\text { rs12447690 } \\
\text { rs7481514 }\end{array}$ & $\begin{array}{l}\text { D: US-Cau } \\
\text { SNP: US-Cau }\end{array}$ & GWAS & $\begin{array}{l}\text { D: } 1117 \\
\text { SNP: } 6469\end{array}$ & $\begin{array}{c}\beta=-5.08, p=0.001 \\
\beta=-6.89, p=1.03 \times 10^{-5} \\
\text { OR }=1.28, p=9.9 \times 10^{-4}\end{array}$ & $\begin{array}{c}\text { Associated with CCT } \\
\text { Associated with reduced CCT } \\
\text { and POAG risk in } \\
\text { low-tension subset }\end{array}$ \\
\hline \multicolumn{8}{|c|}{ Candidate Gene Studies } \\
\hline Chen et al., 2012 [61] & $2 p 16.3$ & rs1533428 & China & SNP & $462 / 577$ & $\mathrm{OR}=2.16, p=0.00025$ & Associated with late-onset POAG \\
\hline Kim et al., 2014 [62] & $10 p 12.31$ & rs7098387 & Korea & SNP & $211 / 904$ & $\mathrm{OR}=2.0, p=0.00038$ & Associated with POAG \\
\hline Fan et al., 2005 [63] & & $\begin{array}{l}\text { rs429358 } \\
\text { rs7412 }\end{array}$ & Japan & SNP & $400 / 281$ & $\mathrm{OR}=0.4, p=0.007$ & $\begin{array}{c}\text { APOE4 confers a protective effect } \\
\text { against NTG }\end{array}$ \\
\hline Lam et al., 2006 [64] & APOE 19q13.2 & $\begin{array}{l}\text { rs } 429358 \\
\text { rs7412 }\end{array}$ & China & SNP & $400 / 300$ & $\mathrm{OR}=0.36, p=0.008$ & $\begin{array}{c}\text { APOE4 confers a protective effect } \\
\text { against NTG }\end{array}$ \\
\hline Lake et al., 2004 [65] & & $\begin{array}{l}\text { rs429358 } \\
\text { rs7412 }\end{array}$ & UK & SNP & $155 / 349$ & $p=\mathrm{ns}$ & None \\
\hline
\end{tabular}


Table 1. Cont

\begin{tabular}{|c|c|c|c|c|c|c|c|}
\hline Studies & Gene/Chromosome & SNP ID & Population * & Study Type & $\begin{array}{c}\text { Study Size } \\
\text { (POAG/Controls) * }\end{array}$ & OR/Beta, $p$ Value & Any Clinical Association * \\
\hline \multicolumn{8}{|c|}{ Candidate Gene Studies } \\
\hline \multirow{3}{*}{ Cao et al., 2012 [66] } & \multirow{8}{*}{ ATOH7 10q21.3-22.1 } & rs7916697 & \multirow{3}{*}{ African-Caribbean } & \multirow{3}{*}{ SNP } & \multirow{3}{*}{$272 / 165$} & $\mathrm{OR}=0.67, p=0.0096$ & $\begin{array}{l}\text { Interacts with rs1063192 near } \\
\text { CDKN2B to reduce POAG risk }\end{array}$ \\
\hline & & rs1900004 & & & & $\mathrm{OR}=1.02, p=0.9076$ & None \\
\hline & & rs 3858145 & & & & $\mathrm{OR}=0.98, p=0.9138$ & None \\
\hline Mabuchi et al., 2012 [67] & & rs1900004 & Japan & SNP & $425 / 191$ & $p=0.028$ & Associated with NTG \\
\hline \multirow[t]{2}{*}{ Chen et al., 2012 [68] } & & rs3858145 & \multirow[t]{2}{*}{ China } & \multirow[t]{2}{*}{ SNP } & \multirow[t]{2}{*}{$142 / 289$} & $\mathrm{OR}=2.69, p<0.05$ & $\begin{array}{l}\text { Showed interaction with RFTN1 } \\
\text { rs690037 }\end{array}$ \\
\hline & & rs61854782 & & & & $\beta=-0.088, p=0.004$ & $\begin{array}{l}\text { Associated with VCDR in controls } \\
\text { but not POAG }\end{array}$ \\
\hline Fan et al., 2011 [69] & & rs1900004 & US-Caucasian & SNP & $539 / 336$ & $\mathrm{OR}=1.89, p=0.025$ & $\begin{array}{c}\text { Associated with increased optic } \\
\text { nerve area }\end{array}$ \\
\hline Dimasi et al., 2012 [70] & & $\begin{array}{l}\text { rs1900004 } \\
\text { rs3858145 }\end{array}$ & $\mathrm{AU}, \mathrm{NZ}$ & SNP & $873 / 886$ & $\begin{array}{l}\mathrm{OR}=1.12^{\prime}, p=0.18 \\
\mathrm{OR}=1.13, p=0.12\end{array}$ & No association \\
\hline \multirow[t]{2}{*}{ Wiggs et al., 2011 [71] } & \multirow{5}{*}{ CAV1/CAV2 7q31.1 } & rs4236601 & \multirow[t]{2}{*}{ US-Caucasian } & \multirow[t]{2}{*}{ SNP } & \multirow[t]{2}{*}{$1000 / 1183$} & $\mathrm{OR}=1.31, p=0.0007$ & $\begin{array}{l}\text { Significantly associated in women } \\
\text { more than men }\end{array}$ \\
\hline & & rs1052990 & & & & $\mathrm{OR}=1.25, p=0.0084$ & $\begin{array}{l}\text { Significantly associated in women; } \\
\text { and nominally associated with NPG } \\
\qquad(p=0.039)\end{array}$ \\
\hline Cao et al., 2012 [66] & & rs4236601 & African-Caribbean & SNP & $272 / 165$ & $\mathrm{OR}=1.15, p=0.3332$ & No association \\
\hline \multirow[t]{2}{*}{ Loomis et al., 2014 [72] } & & rs4236601 & \multirow[t]{2}{*}{ US-Caucasian } & \multirow[t]{2}{*}{ SNP } & \multirow[t]{2}{*}{$\begin{array}{l}\text { R1: } 976 / 2132 \\
\text { R2: } 1140 / 2290\end{array}$} & $\begin{aligned} p_{\text {meta }}= & 2.61 \times 10^{(-7)}, p_{\text {women }}= \\
& 1.59 \times 10^{(-5)}\end{aligned}$ & $\begin{array}{l}\text { Associated with early paracentral } \\
\text { VF defect }\end{array}$ \\
\hline & & rs17588172 & & & & $p_{\text {meta }}=1.07 \times 10^{(-4)}$ & $\begin{array}{l}\text { Associated with early paracentral } \\
\text { VF defect }\end{array}$ \\
\hline Kuehn et al., 2011 [73] & & rs4236601 & US & SNP & $545 / 297$ & $p=0.5$ & No association \\
\hline Cao et al., 2012 [66] & \multirow{3}{*}{$\begin{array}{c}\text { CARD10 22q13.1 } \\
\text { CDC7/TGFBR3 1p22 }\end{array}$} & rs9607469 & African-Caribbean & SNP & $272 / 165$ & $\mathrm{OR}=1.13, p=0.5096$ & No association \\
\hline Cao et al., 2012 [66] & & rs1192415 & African-Caribbean & SNP & $272 / 165$ & $\mathrm{OR}=1.14, p=0.4802$ & No association \\
\hline Dimasi et al., 2012 [70] & & rs1192415 & $\mathrm{AU}, \mathrm{NZ}$ & SNP & $873 / 886$ & $\mathrm{OR}=1.22, p=0.03$ & $\begin{array}{l}\text { Showed nominal significance with } \\
\text { optic disc area }\end{array}$ \\
\hline \multirow{2}{*}{ Cao et al., 2012 [66] } & \multirow{6}{*}{ CDKN2B (-AS1) 9p21 } & rs1063192 & \multirow{2}{*}{ African-Caribbean } & \multirow{2}{*}{ SNP } & \multirow[t]{2}{*}{$272 / 165$} & $\mathrm{OR}=0.39, p=0.0008$ & $\begin{array}{l}\text { Minor allele was protective } \\
\text { against POAG }\end{array}$ \\
\hline & & rs4977756 & & & & $\mathrm{OR}=0.89, p=0.4507$ & No association \\
\hline Fan et al., 2011 [69] & & rs1063192 & US-Caucasian & SNP & $539 / 336$ & $\mathrm{OR}=0.73, p=0.0006$ & $\begin{array}{c}\text { Associated with decreased VCDR } \\
\text { and POAG risk }\end{array}$ \\
\hline Mabuchi et al., 2012 [67] & & rs1063192 & Japan & SNP & $425 / 191$ & $\beta=0.11, p=0.0043$ & $\begin{array}{l}\text { Associated with VCDR; } \\
\text { and NTG }(p=0.023)\end{array}$ \\
\hline Dimasi et al., 2012 [70] & & rs1063192 & $\mathrm{AU}, \mathrm{NZ}$ & SNP & $873 / 886$ & $\mathrm{OR}=0.74, p=2.2 \times 10^{-5}$ & $\begin{array}{l}\text { More strongly associated with } \\
\text { advanced open-angle glaucoma }\end{array}$ \\
\hline Burdon et al., 2012 [74] & & $\begin{array}{l}\text { rs10120688 } \\
\text { rs7049105 }\end{array}$ & $\mathrm{AU}, \mathrm{NZ}$ & $\mathrm{SNP}$ & $1432 / 595$ & $\begin{array}{l}\mathrm{VCDR}-\beta=0.016, p=0.03 \\
\mathrm{IOP}-\beta=-2.135, p=0.001\end{array}$ & $\begin{array}{c}\text { Associated with larger VCDR and } \\
\text { lower IOP }\end{array}$ \\
\hline
\end{tabular}


Table 1. Cont

\begin{tabular}{|c|c|c|c|c|c|c|c|}
\hline Studies & Gene/Chromosome & SNP ID & Population * & Study Type & $\begin{array}{c}\text { Study Size } \\
\text { (POAG/Controls) * }\end{array}$ & OR/Beta, $p$ Value & Any Clinical Association * \\
\hline \multicolumn{8}{|c|}{ Candidate Gene Studies } \\
\hline Mabuchi et al., 2012 [67] & \multirow{2}{*}{ CHEK2 22q12.1 } & rs1547014 & Japan & SNP & $425 / 191$ & $\beta=0.11, p=0.0079$ & \multirow{2}{*}{$\begin{array}{l}\text { Associated with VCDR; } \\
\text { and HTG }(p=0.013) \\
\text { No association }\end{array}$} \\
\hline Dimasi et al., 2012 [70] & & rs1547014 & $\mathrm{AU}, \mathrm{NZ}$ & SNP & $873 / 886$ & $\mathrm{OR}=0.98, p=0.77$ & \\
\hline Dimasi et al., 2012 [70] & $\begin{array}{l}\text { COL5A1/RXRA } \\
9 q 34.2-q 34.3 \\
\end{array}$ & $\begin{array}{l}\text { rs1536482 } \\
\text { rs7044529 } \\
\end{array}$ & $\mathrm{AU}, \mathrm{NZ}$ & SNP & $873 / 886$ & $\begin{array}{l}\mathrm{OR}=0.94, p=0.46 \\
\mathrm{OR}=1.00, p=0.98\end{array}$ & No association \\
\hline Desronvil et al., 2010 [75] & COL8A2 1p34.2 & rs274754 & US-Caucasian & SNP & 100 & $p=0.018$ & Associated with corneal thickness \\
\hline Dimasi et al., 2010 [76] & FBN1 15q21.1 & rs17352842 & AU-Caucasian & SNP & 956 & $p=0.02$ & Associated with CCT \\
\hline Rocha et al., 2011 [77] & \multirow{4}{*}{ GSTT1/GSTM1 1p13.3 } & null $>$ positive & Brazil & SNP & $87 / 85$ & $\mathrm{OR}=2.4, p=0.016$ & $\begin{array}{l}\text { T1M0 genotype associated with } \\
\text { higher IOP and severe defect of right } \\
\text { eye optic nerve and visual field }\end{array}$ \\
\hline Juronen et al., 2000 [78] & & positive $>$ null & Estonia & SNP & $250 / 202$ & $\mathrm{OR}=1.83, p=0.002$ & $\begin{array}{l}\text { GSTM1 were at significant risk for } \\
\text { glaucoma and even higher in } \\
\text { smokers (OR }=3.86, p=0.012)\end{array}$ \\
\hline Jansson et al., 2003 [79] & & positive $=$ null & Sweden & SNP & $200 / 200$ & $p=\mathrm{ns}$ & No association \\
\hline Fan et al., 2010 [80] & & positive $=$ null & China & SNP & $405 / 201$ & $p=\mathrm{ns}$ & No association \\
\hline Liu et al., 2010 [81] & \multirow{4}{*}{ NTF4 19q13.33 } & \multirow{4}{*}{$\begin{array}{l}\text { Whole gene } \\
\text { Whole gene } \\
\text { c.338T }>\mathrm{C} \\
\text { c. } 470 \mathrm{G}>\mathrm{C} \\
\text { c. } 545 \mathrm{C}>\mathrm{T}\end{array}$} & US-Caucasian & SNP & $443 / 533$ & - & \multirow{4}{*}{$\begin{array}{c}\text { Not associated with POAG } \\
\text { No association } \\
\text { Rare cause of POAG in Chinese } \\
\text { May be a rare cause of POAG }\end{array}$} \\
\hline Rao et al., 2010 [82] & & & India & SNP & $141 / 285$ & $p=0.2$ & \\
\hline Vithana et al., 2010 [83] & & & China & SNP & $174 / 91$ & - & \\
\hline Chen et al., 2012 [84] & & & China & $\mathrm{SNP}$ & $720 / 230$ & - & \\
\hline Aung et al., 2002 [85] & \multirow[t]{4}{*}{ OPA1 3q28-q29 } & $\begin{array}{l}\text { rs166850 } \\
\text { rs10451941 }\end{array}$ & UK & SNP & $163 / 86$ & $\begin{array}{c}\mathrm{OR}=3.1, p=0.002 \\
p=0.03\end{array}$ & \multirow{2}{*}{$\begin{array}{l}\text { rs166850 combined with rs10451941 } \\
\text { was more strongly associated with } \\
\text { NTG ( } p=0.00001) \\
\text { Increased risk of NTG; and age at } \\
\text { diagnosis in HTG }(p=0.048)\end{array}$} \\
\hline Mabuchi et al., 2007 [86] & & rs10451941 & Japan & SNP & $285 / 185$ & $\mathrm{OR}=2.27, p=0.004$ & \\
\hline Yao et al., 2006 [87] & & $\begin{array}{l}\text { rs166850 } \\
\text { rs10451941 }\end{array}$ & African-Caribbean & SNP & $109 / 48$ & $p=\mathrm{ns}$ & No association \\
\hline Fan et al., 2010 [80] & & $\begin{array}{l}\text { rs166850 } \\
\text { rs10451941 }\end{array}$ & China & SNP & $405 / 201$ & $p=\mathrm{ns}$ & No association \\
\hline Dimasi et al., 2010 [76] & PAX6 11p13 & rs3026398 & AU-Caucasian & SNP & 956 & $p=0.02$ & $\begin{array}{l}\text { Associated with CCT; more strongly } \\
\text { with rs662702 haplotype }(p=0.009)\end{array}$ \\
\hline Chen et al., 2012 [61] & \multirow{2}{*}{ PLXDC2 10p12.31 } & rs7081455 & China & SNP & $462 / 577$ & $\mathrm{OR}=1.25, p=0.31$ & No association \\
\hline Cao et al., 2012 [66] & & rs7081455 & African-Caribbean & SNP & $272 / 165$ & $\mathrm{OR}=1.04, p=0.8052$ & No association \\
\hline Chen et al., 2012 [68] & RFTN1 3p24.3 & rs3858145 & China & SNP & $142 / 289$ & $\beta=25.66, p=0.029$ & Associated with CCT \\
\hline
\end{tabular}


Table 1. Cont

\begin{tabular}{|c|c|c|c|c|c|c|c|}
\hline Studies & Gene/Chromosome & SNP ID & Population * & Study Type & $\begin{array}{c}\text { Study Size } \\
\text { (POAG/Controls) * }\end{array}$ & OR/Beta, $p$ Value & Any Clinical Association * \\
\hline \multicolumn{8}{|c|}{ Candidate Gene Studies } \\
\hline Fan et al., 2011 [69] & \multirow{6}{*}{ SIX1/SIX6 14p22-23 } & rs10483727 & US-Caucasian & $\mathrm{SNP}$ & $539 / 336$ & $\mathrm{OR}=1.33, p=0.0043$ & $\begin{array}{l}\text { Associated with increased VCDR } \\
\text { and POAG risk }\end{array}$ \\
\hline Dimasi et al., 2012 [70] & & rs10483727 & $\mathrm{AU}, \mathrm{NZ}$ & SNP & $873 / 886$ & $\mathrm{OR}=1.38, p=6.2 \times 10^{-6}$ & $\begin{array}{l}\text { Strongly associated with } \\
\text { open-angle glaucoma }\end{array}$ \\
\hline Cao et al., 2012 [66] & & rs10483727 & African-Caribbean & SNP & $272 / 165$ & $\mathrm{OR}=0.77, p=0.4151$ & No association \\
\hline Mabuchi et al., 2012 [67] & & rs10483727 & Japan & SNP & $425 / 191$ & $p=0.017$ & $\begin{array}{c}\text { Associated with age at } \\
\text { diagnosis in NTG }\end{array}$ \\
\hline \multirow{2}{*}{ Carnes et al., 2014 [88] } & & rs10483727 & \multirow{2}{*}{ US-Caucasians } & SNP & \multirow{2}{*}{$262 / 256$} & $\mathrm{OR}=1.32, p=3.87 \times 10^{-11}$ & Significantly associated with POAG \\
\hline & & rs33912345 & & SNP & & $\mathrm{OR}=1.27, p=4.2 \times 10^{-10}$ & $\begin{array}{l}\text { Associated with POAG; and } \\
\text { thickness of retinal nerve fiber layer }\end{array}$ \\
\hline Mabuchi et al., 2011 [89] & \multirow{2}{*}{$S R B D 12 p 21$} & rs3213787 & Japan & SNP & $370 / 191$ & $\begin{array}{c}p=0.0003 \text { in NTG and } p= \\
0.0013 \text { in HTG }\end{array}$ & $\begin{array}{c}\text { Associated with HTG and NTG } \\
\text { including late-onset }\end{array}$ \\
\hline Cao et al., 2012 [66] & & rs3213787 & African-Caribbean & SNP & $272 / 165$ & $\mathrm{OR}=0.45, p=0.2882$ & None \\
\hline Takano et al., 2012 [90] & \multirow{4}{*}{ TLR4 9q33.1 } & rs2149356 & Japan & SNP & $449 / 107$ & $p=0.000058$ & Associated with NTG \\
\hline Chen et al., 2012 [61] & & rs7037117 & China & SNP & $462 / 577$ & $\mathrm{OR}^{\prime}=0.99, p=0.99$ & $\begin{array}{c}\text { No association } \\
\text { 1.47- to } 1.65 \text {-fold increased risk of }\end{array}$ \\
\hline Shibuya et al., 2008 [91] & & rs7037117 & Japan & SNP & $215 / 318$ & $p=0.0095$ & $\begin{array}{l}\text { NTG; strongest association with } \\
\text { rs10759930 haplotype }\end{array}$ \\
\hline Cao et al., 2012 [66] & & rs7037117 & African-Caribbean & SNP & $272 / 165$ & $\mathrm{OR}=0.73, p=0.0571$ & No association \\
\hline $\begin{array}{l}\text { Sharma et al., } 2012 \text { [92] } \\
\text { Ozel et al., } 2014 \text { [93] }\end{array}$ & TMCO1 1q24 & $\begin{array}{l}\text { rs4656461 } \\
\text { rs7518099 }\end{array}$ & $\begin{array}{c}\text { AU, NZ } \\
\text { US-Caucasian }\end{array}$ & $\begin{array}{l}\text { SNP } \\
\text { SNP }\end{array}$ & $\begin{array}{l}1420 \\
6.236\end{array}$ & $\begin{array}{c}\beta=-2.56, p=0.004 \\
\quad p=8 \times 10^{-8}\end{array}$ & $\begin{array}{l}\text { Correlation with age at diagnosis } \\
\text { Strongly associated with IOP }\end{array}$ \\
\hline $\begin{array}{l}\text { Chen et al., } 2012 \text { [61] } \\
\text { Cao et al., } 2012 \text { [66] }\end{array}$ & TMTC2 12q21.31 & $\begin{array}{l}\text { rs7961953 } \\
\text { rs7961953 }\end{array}$ & $\begin{array}{c}\text { China } \\
\text { African-Caribbean }\end{array}$ & $\begin{array}{l}\text { SNP } \\
\text { SNP }\end{array}$ & $\begin{array}{l}462 / 577 \\
272 / 165\end{array}$ & $\begin{array}{c}\mathrm{OR}=1.15, p=0.35 \\
\mathrm{OR}=0.89, p=0.5559\end{array}$ & $\begin{array}{l}\text { No association } \\
\text { No association }\end{array}$ \\
\hline Fan et al., 2010 [80] & \multirow{3}{*}{$T N F \propto 6 p 21.3$} & \multirow{3}{*}{$\begin{array}{c}\text { rs1800629 } \\
\text { rs4645836 } \\
\text { rs1800629 } \\
\text { rs361525 }\end{array}$} & China & SNP & $405 / 201$ & \multirow{3}{*}{$\begin{array}{c}p=0.012 \\
\mathrm{OR}=0.63, p=0.017 \\
\mathrm{OR}=0.96, p>0.05 \\
\mathrm{OR}=0.52, p>0.05\end{array}$} & Associated with HTG \\
\hline Wang et al., 2012 [94] & & & China & SNP & $234 / 230$ & & \multirow[b]{2}{*}{ Not associated among Caucasian } \\
\hline Mossböck et al., 2006 [95] & & & $\mathrm{AU}$ & SNP & $114 / 228$ & & \\
\hline Rao et al., 2010 [82] & $\begin{array}{l}\text { VAV2 9q34.1 } \\
\text { VAV3 1p13.3 }\end{array}$ & $\begin{array}{l}\text { rs2156323 } \\
\text { rs2801219 }\end{array}$ & India & SNP & $141 / 285$ & $\begin{array}{l}p=0.533 \\
p=0.133\end{array}$ & No association \\
\hline Dimasi et al., 2012 [70] & ZNF469 16q24 & $\begin{array}{c}\text { rs12447690 } \\
\text { rs9938149 }\end{array}$ & $\mathrm{AU}, \mathrm{NZ}$ & SNP & $873 / 886$ & $\begin{array}{l}\mathrm{OR}=1.01, p=0.91 \\
\mathrm{OR}=0.94, p=0.46\end{array}$ & No association \\
\hline Chen et al., 2012 [61] & & rs693421 & China & SNP & $462 / 577$ & $\mathrm{OR}=0.98, p=0.31$ & No association \\
\hline Cao et al., 2012 [66] & ZP4 1q43 & rs547984 & African-Caribbean & SNP & $272 / 165$ & $\mathrm{OR}=1.05, p=0.7374$ & No association \\
\hline Kim et al., 2014 [62] & & rs693421 & Korea & SNP & $211 / 904$ & $\mathrm{OR}=1.4, p=0.0082$ & Associated with POAG \\
\hline
\end{tabular}


Table 1. Cont

\begin{tabular}{|c|c|c|c|c|c|c|c|}
\hline Studies & Gene/Chromosome & SNP ID & Population * & Study Type & $\begin{array}{c}\text { Study Size } \\
\text { (POAG/Controls) * }\end{array}$ & OR/Beta, $p$ Value & Any Clinical Association * \\
\hline \multicolumn{8}{|c|}{ Candidate Gene Studies } \\
\hline Li et al., 2015 [48] & $\begin{array}{c}\text { CDKN2B-AS1 9p21 } \\
\text { CDC7-TGFBR3 1p22 } \\
\text { FNDC3B 3q25.31 }\end{array}$ & $\begin{array}{l}\text { rs2157719 } \\
\text { rs1192415 } \\
\text { rs } 4894796\end{array}$ & Saudi Arabia & SNP & $R^{* *}: 236 / 655$ & $\begin{array}{l}\mathrm{OR}=1.24, p=0.146 \\
\mathrm{OR}=1.24, p=0.146 \\
\mathrm{OR}=1.03, p=0.779\end{array}$ & $\begin{array}{l}- \\
- \\
-\end{array}$ \\
\hline Neamatzadeh et al., 2015 [96] & TP53 17p13.1 & rs1042522 & Iranian & $\mathrm{SNP}$ & $65 / 65$ & $\mathrm{OR}=2.1, p<0.05$ & $\begin{array}{c}\text { Pro72 allele is associated with POAG } \\
\text { risk }\end{array}$ \\
\hline Emam et al., 2014 [97] & NOS3 7q36 & $\begin{array}{c}\text { rs2070744 } \\
\text { rs1799983 } \\
27 \mathrm{bp}-\mathrm{VNTR}-\mathrm{a} / \mathrm{b}\end{array}$ & Egypt & SNP & $\begin{array}{c}160 / 110 \\
- \\
-\end{array}$ & $\begin{array}{c}\mathrm{OR}=1.86, p<0.0001 \\
\mathrm{OR}=1.28, p=0.21 \\
\mathrm{OR}=0.81, p=0.33\end{array}$ & $\begin{array}{c}\text { rs2070744 is associated with high } \\
\text { tension glaucoma; and with plasma } \\
\text { nitrite/nitrate levels }(p<0.001) \\
- \\
-\end{array}$ \\
\hline Abu-Amero et al., 2013 [98] & CAT 11p13 & rs1001179 & Saudi Arabia & SNP & $225 / 403$ & $\mathrm{OR}=0.81, p=0.218$ & $\begin{array}{l}\text { Associated with age of onset, } \\
\text { and trend towards IOP, } \\
\text { and duration of glaucoma }\end{array}$ \\
\hline Abu-Amero et al., 2014 [99] & SOD2 6q25.3 & rs 4880 & Saudi Arabia & SNP & $226 / 403$ & $\mathrm{OR}=1.0, p=0.988$ & Trend towards age of onset and IOP \\
\hline Abu-Amero et al., 2012 [100] & CAV1/CAV2 7q31 & rs4236601 & Saudi Arabia & SNP & $220 / 405$ & $\mathrm{OR}=1.06, p=0.699$ & - \\
\hline Abu-Amero et al., 2012 [101] & LOXL1 15q24.1 & $\begin{array}{l}\text { rs1048661 } \\
\text { rs3825942 } \\
\text { rs2165241 }\end{array}$ & Saudi Arabia & SNP & $\begin{array}{c}96 / 101 \\
- \\
-\end{array}$ & $\begin{array}{l}p=0.866 \\
p=0.477 \\
p=0.176\end{array}$ & $\begin{array}{l}- \\
- \\
-\end{array}$ \\
\hline Abu-Amero et al., 2006 [102] & $\begin{array}{l}\text { MYOC 1q24.3 } \\
\text { OPTN } 10 p 13\end{array}$ & $\begin{array}{c}22259 \mathrm{G} / \mathrm{T} \\
(\mathrm{G} 324 \mathrm{~V}) \\
412 \mathrm{G} / \mathrm{A}(\mathrm{T} 34 \mathrm{~T}) \\
469 \mathrm{G} / \mathrm{C}(\mathrm{Q} 53 \mathrm{H})\end{array}$ & Saudi Arabia & SNP & $\begin{array}{c}27 / 96 \\
- \\
-\end{array}$ & $\begin{array}{l}p=0.74 \\
p=0.61 \\
p=0.28\end{array}$ & $\begin{array}{l}- \\
- \\
-\end{array}$ \\
\hline $\begin{array}{c}\text { Zanon-Moreno et al., } 2013 \\
\text { [103] }\end{array}$ & $\begin{array}{c}\text { TTPA 8q12.3 } \\
\text { SEC14L2/TAP 22q12.2 } \\
\text { GPX4 19p13.3 }\end{array}$ & $\begin{array}{l}\text { rs6994076 } \\
\text { rs737723 } \\
\text { rs757228 }\end{array}$ & Mediterranean & SNP & $\begin{array}{l}- \\
- \\
-\end{array}$ & $\begin{array}{l}\mathrm{OR}=2.47, p<0.001 \\
\mathrm{OR}=1.38, p=0.122 \\
\mathrm{OR}=2.24, p<0.001 \\
\mathrm{OR}=0.80, p=0.337\end{array}$ & $\begin{array}{c}\text { Associated with POAG risk; and } \\
\text { plasma vitamin C levels }(p<0.001) \\
\text { Associated with plasma vitamin E } \\
\text { levels }(p<0.001) \\
\text { Associated with POAG risk; and } \\
\text { nominal ( } p=0.047) \text { gene-gene } \\
\text { interaction with SNP rs1279683 } \\
\text { - }\end{array}$ \\
\hline
\end{tabular}


Table 1. Cont

\begin{tabular}{|c|c|c|c|c|c|c|c|}
\hline Studies & Gene/Chromosome & SNP ID & Population * & Study Type & $\begin{array}{c}\text { Study Size } \\
\text { (POAG/Controls) * }\end{array}$ & OR/Beta, $p$ Value & Any Clinical Association * \\
\hline \multicolumn{8}{|c|}{ Candidate Gene Studies } \\
\hline \multirow{4}{*}{$\begin{array}{c}\text { Zanon-Moreno et al., } 2011 \\
\text { [104] }\end{array}$} & & rs176990 & \multirow{4}{*}{ Mediterranean } & \multirow{4}{*}{ SNP } & $150 / 150$ & $\mathrm{OR}=0.97, p=0.826$ & - \\
\hline & & rs190910 & & & - & $\mathrm{OR}=0.83, p=0.315$ & - \\
\hline & SLC23A1 5q31.2 & rs10063949 & & & - & $\mathrm{OR}=1.19, p=0.552$ & - \\
\hline & SLC23A2 20p13 & rs1279683 & & & - & $\mathrm{OR}=1.67, p=0.010$ & $\begin{array}{l}\text { Associated with POAG risk; and } \\
\text { plasma vitamin C levels }(p<0.001)\end{array}$ \\
\hline \multirow{3}{*}{ Abu-Amero et al., 2008 [105] } & \multirow{3}{*}{ GSTT1/GSTM1 1p13.3 } & T0M0 & \multirow{3}{*}{ Saudi Arabia } & \multirow{3}{*}{ SNP } & $49 / 120$ & $\mathrm{OR}=5.67, p=0.06$ & $\begin{array}{l}\text { GSTT1 and GSTM1 positive } \\
\text { genotypes are at risk for POAG }\end{array}$ \\
\hline & & T1M0 & & & - & $\mathrm{OR}=10.2, p=0.00001$ & - \\
\hline & & T0M1 & & & - & $\mathrm{OR}=11.3, p=0.00001$ & - \\
\hline Unal et al., 2007 [106] & GSTT1/GSTM1 1p13.3 & T0M1 & Turkey & SNP & $144 / 121$ & $\mathrm{OR}=3.46, p<0.005$ & $\begin{array}{l}\text { GSTM1 positive and GSTT1 null } \\
\text { genotypes are associated with } \\
\text { increased risk of POAG }\end{array}$ \\
\hline Al-Dabbagh et al. [107] & APOE 19q13.2 & $\begin{array}{l}\text { rs429358 } \\
\text { rs7412 }\end{array}$ & Saudi Arabia & SNP & $60 / 130$ & $\mathrm{OR}=2.75, p=0.034$ & $\begin{array}{l}\text { APOE4 allele is a risk factor for } \\
\text { POAG }\end{array}$ \\
\hline \multirow{2}{*}{ Saglar et al., 2009 [108] } & APOE 19q13.2 & $\begin{array}{l}\text { rs429358 } \\
\text { rs7412 }\end{array}$ & \multirow{2}{*}{ Turkey } & \multirow{2}{*}{ SNP } & $75 / 119$ & $p=0.38$ & - \\
\hline & TP53 17p & rs 1042522 & & & - & $p=0.12$ & - \\
\hline Nilforoushan et al. [109] & MTHFR 1p36.3 & rs1801133 & Iran & - & $73 / 90$ & $p=0.337$ & - \\
\hline
\end{tabular}




\section{GWAS and POAG}

Nakano et al. described the first GWAS in the Japanese POAG population with patients predominantly having NTG [38]. This was a two-stage GWAS involving a discovery cohort and a replication cohort. The study reported significant loci on chromosomes 1,10 and 12 that included genes such as ZP4, PLXDC2 and TMCT2 (DKFZp762A217), respectively. However, none of the SNPs achieved a genome-wide significance $\left(p<5 \times 10^{-8}\right)$ even in the combined analysis and, therefore, they await further evaluation in additional cohorts. Meguro et al. reported the first genome-wide significant $\left(p=2.5 \times 10^{-9}\right.$, odds ratio $\left.(\mathrm{OR})=2.80\right)$ association for SNP rs3213787 in SRBD1 in the Japanese NTG population [39]. Two other studies have replicated this finding in a Japanese NTG and high-tension glaucoma (HTG) cohort [89] and a US Caucasian POAG cohort [110], but not in the African-Caribbean cohort [66].

GWASs have been able to identify certain common variants that are of significance to the understanding of POAG pathogenesis. These include SNPs near CAV1 and CAV2 in an Icelandic cohort [40], in TMCO1 and CDKN2B-AS1 in an Australian cohort [41], in CDKN2B-AS1, SIX1/SIX6, and the 8q22 locus in Europeans [42], in GAS7 and TMCO1 in US Caucasians [49], and in CDKN2B-AS1, CDC7/TGFBR3 and FNDC3B in Asian, African and European cohorts [48].

The caveolin genes have been postulated to influence transforming growth factor-beta (TGF- $\beta$ ) or nitric oxide signaling pathways involved in POAG pathogenesis. The locus on chromosome 7q31 has been studies in US Caucasians, Africans, and the Saudi Arabian population with inconsistent results $[40,66,71-73,100]$. A recent meta-analysis of five studies, including 5774 POAG cases and 40,598 healthy controls, suggested that SNP rs4236601 is associated with POAG risk in Caucasian and Asian populations but not in African and Saudi populations [111]. Australian GWAS identified two loci, TMCO1 (1q24) and CDKN2B-AS1 (9p21), to be associated with advanced glaucoma. The association of the TMCO1 locus with POAG has been replicated in another GWAS for a Caucasian cohort [49], and associated with increase in IOP as well [49,93]; the carriers of risk alleles for SNP rs4656461 have been reported to be associated with a younger age at diagnosis [92]. The ciliary body, trabecular meshwork and retina show abundant TMCO1 expression. However, its precise role in POAG pathogenesis is unclear. So far, there are no published reports of association studies at the TMCO1 locus in the Middle East population.

Since the identification of the association between the CDKN2B/CDKN2B-AS1 locus and POAG in the Australian cohort, several GWASs have replicated this association in the US Caucasian [42], Japanese [43-45], Asian, African, and European populations [48], providing strong evidence for the association of this locus with POAG. In addition, many studies have reported a positive association of SNPs in CDKN2B in several other populations using a candidate-gene approach $[66,67,69,70,74]$. These SNPs are located in an anti-sense non-protein coding gene, CDKN2BAS, within the CDKN2A/B gene cluster. CDKN2B is a tumor suppressor gene and, with its suggested role in the TGF- $\beta$ pathway, may play a critical role in glaucoma pathogenesis [112,113]. Interestingly, carriers of the CDKN2B-AS1 risk alleles are associated with larger VCDR $[53,54]$ and low IOP as compared to the wild-type carriers [74]. On the basis of these findings, it has been suggested that the $C D K N 2 B / C D K N 2 B-A S 1$ locus of 9p21 may possibly predispose a person to glaucomatous optic neuropathy in a mechanism that may not be dependent on IOP and highlights the importance of the chromosome 9p21 susceptibility locus as a risk factor in the development of POAG [114].

Recently, Li et al. performed a GWAS on 3504 POAG cases and 9746 controls. The positive significant findings of this phase were then replicated in 9173 POAG cases and 26,780 controls across 18 different collections of Asian, African, and European populations including a replication cohort from our center in Saudi Arabia [48]. The study confirmed and provided strong evidence of an association at the CDKN2B-AS1 locus (rs2157719, OR $=0.71, p=2.81 \times 10^{-33}$ ), and also identified SNP rs1192415 in the CDC7-TGFBR3 gene (1p22) showing significant association with POAG (OR $\left.=1.13, p=1.60 \times 10^{-8}\right)$ in the Asian, African and European populations, as well as SNP rs4894796 in FNDC3B (3q25.31) showing a significant association in Asians only $\left(\mathrm{OR}=0.89, p=7.93 \times 10^{-8}\right)$. 
Interestingly, these results were found to be non-significant in the Saudi replication cohort, indicating that the genetic cause for POAG in the Saudi population may be different than those from Asian, African and European descent.

GWAS studies by Wiggs et al. and Osman et al. in the Caucasian POAG and Japanese POAG cases, respectively, have demonstrated a strong association of SNP rs10483727 located in the in the intergenic region between the SIX1 and SIX6 locus (14q23) [42,43]. SIX6 has been shown to express in the developing and adult human retina [115]. Moreover, the association of SNP rs10483727 in the SIX1/SIX6 region has also been replicated in other Caucasian POAG cohorts $[67,69,70,88]$ but not in the African-Caribbean subjects [66]. After the association of the CDKN2B-AS1 region on chromosome 9p21, the second most consistent association with POAG has been observed in the SIX1/SIX6 locus and so it would be interesting to know if this locus is associated with POAG in the Saudi or other Middle Eastern populations. However, currently there are no published reports of association of SIX1/SIX6 locus with POAG in the middle-east population.

Recently, 11p11.2 (containing multiple genes), ABCA1, ABO, AFAP1, ARHGEF12, FAR2, GGA3, GMDS, PKDREJ, and PMM2 were added to the newly discovered genes associated with POAG [46,47,50-52]. These variants were significantly associated with glaucoma and the related functional visual field loss that could make them future study targets for glaucoma patients in the Middle East.

\section{GWAS and Quantitative Endophenotype Traits}

The genetic evaluation of quantitative endophenotype traits is often very useful in complex multifactorial diseases to understand the contribution of specific traits to the overall disease phenotype. A similar strategy has been successfully used in POAG to understand the contribution of proposed endophenotypes including IOP, VCDR, optic disc area and CCT to the overall disease process. GWASs have been performed to examine the genetic components of these endophenotypes in POAG and the normal population. van Koolwijk and colleagues performed a GWAS for IOP in POAG patients of European descent and identified SNPs rs11656696 and rs7555523, located in GAS7 and TMCO1, respectively, suggesting a role for these two genes in IOP regulation [49]. Other loci found to be associated with IOP so far include FNDC3B, ABCA1, ABO, 11p11.2, ARHGEF12 [50,52]. Another three loci, FAR2, GGA3, and PKDREJ, did not reach a genome-wide significance level $\left(p<10^{-5}\right)$ [51]. Three independent GWASs have evaluated the association of optic disc parameters (VCDR and optic disc area) in the normal general population. The loci associated included ATOH7, CDC7/TGFBR3 and SALL1, CARD10 for the optic disc area, and CDKN2B, SIX1, SCYL1/LTBP3, CHEK2, and DCLK1, in addition to ATOH7, for the VCDR [53-56]. An exome sequencing also reported the SIX6 locus to influence $\operatorname{VCDR}\left(p=7.74 \times 10^{-7}\right)$ [57]. A subsequent meta-analysis of the Rotterdam study with the Twin UK study [54] demonstrated a strong association of ATOH7, CDKN2B, and SIX1 in POAG with borderline association for CDC7/TGFBR3 and SALL4 (both $p=0.04)$. CARD10 was not found to be associated with African-Caribbean POAG cases [66], whereas CHEK2 was reported to be associated with VCDR and HTG among the Japanese [67] but not in Europeans [70]. Moreover, multiple studies have provided strong evidence of association of ATOH7 [66-69] CDKN2B(-AS1) [66,67,69,70,74] and SIX1/SIX6 [69,70] with POAG. CCT is an important risk factor for POAG in individuals with increased IOP, and over 26 loci have been reported [116]. GWASs have identified several loci associated with CCT in the normal general population (Asian and European descent) and POAG cases (US Caucasians). These loci include ZNF469, COL5A1, AKAP13, AVGR8, and COL8A2 [58-60]. The ZNF469 and COL5A1 loci have been found to be associated with CCT in both the Caucasian and Asian cohorts $[58,59]$.

The possible role of these newly discovered loci associated with POAG and its endophenotypes in understanding the pathophysiology of POAG has been elegantly reviewed by Iglesias et al. elsewhere [117]. The review integrates current knowledge in POAG from human and experimental data and dissects the contribution of the newly discovered genetic loci with the known molecular and biological processes, including extracellular matrix remodeling; TGF- $\beta$ and tumor necrosis factor 
$\alpha(\mathrm{TNF}-\alpha)$ signaling; and the vascular tone pathway, that have been implicated in the pathogenesis of POAG.

\section{Candidate Genes and POAG}

Recent reviews by Takamota and Araie [32] and Janssen et al. [31] presented a list of genes identified from numerous GWAS and association studies thus far. Taken together, the list of almost 50 genes may represent highly likely candidate genes that may be involved in POAG pathogenesis. Many studies have been performed to replicate the GWAS findings in the Asian, African-Caribbean and Caucasian/European populations using the candidate-gene approach $[61,66,67,69-76,84,89,92,93]$. Also, many studies were performed to test the association of specific known genes/SNPs with POAG using the same approach in different populations including Middle Eastern [61-66,76-87,90,91,94-109]. These SNP replication and genetic association studies in the Middle Eastern and other populations are also listed in Table 1. Among these, consistent findings have been reported for ATOH7 [66-69], CDKN2B (-AS1) $[66,67,69,70,74]$, GSTT1/GSTM1 [77,78,105,106], SIX1/SIX6 [69,70] and TMCO1 [92,93] loci, indicating a potential role of these genes/loci in the pathogenesis of POAG. However, except for the glutathione-S transferase (GST) polymorphism, none of these loci have been either found to be associated with POAG (e.g., CAV1/CAV2, CDC7/TGFBR3, FNDC3) or the association has not been reported yet (e.g., $A T O H 7, C D K N 2 B(-A S 1), S I X 1 / S I X 6$, TMCO1) in the Middle Eastern population. However, the positive findings of GSTT1 and GSTM1 genotypes in the Middle Eastern population may be very interesting, highlighting the role of anti-oxidants and/or oxidative stress-related pathways/mechanisms in the pathogenesis of POAG in this population. This view is strongly supported by recent meta-analysis studies that examined the association of GST polymorphisms and the risk of POAG [118-120]. We have previously studied SNPs in two of the anti-oxidant genes, CAT (rs1001179) and SOD2 (rs4880) [98,99], in the Saudi POAG patients. However, the studies did not provide any direct association with POAG but indicated a trend towards an association with IOP and age of onset of POAG. In addition, some studies have demonstrated moderate evidence for association of SNPs in TP53, NOS3, SEC14L2/TAP, and APOE [96,97,103,107]. However, these studies have been limited by sample size and would need further investigations in a large population-based cohort. The examination of causative genes such as MYOC, OPTN and LOXL1 in Saudi POAG cases has also provided negative results [102,105]. Table 2 list all genes associated with POAG and their possible role in POAG pathogenesis.

\section{Final Remarks}

There is significant progress in understanding the genetic basis of POAG, largely due to the application of GWAS methodology in different populations. In recent years, GWASs have identified several loci associated with POAG including CAV1/CAV2, TMCO1, CDKN2B-AS1, CDC7-TGFBR3, SIX1/SIX6, GAS7 and ATOH7.

The association between the CDKN2B(-AS1) locus on chromosome 9p21 and POAG has been extensively established across different populations and represents a major genetic risk factor for POAG. Studies involving the SIX1/SIX6 and the ATOH7 loci affecting the optic disc parameters and POAG itself have also been reproducible. Other loci seem to be more ethnicity-specific. CAV1/CAV2 and CDC7-TGFBR3 loci do not seem to contribute to POAG in the Middle East and the role of other newly discovered loci is yet to be established. Moreover, the GSTT1/GSTM1 genotypes were found to be strongly associated with POAG in the Middle Eastern population and more studies may be needed to examine the role of oxidative stress and anti-oxidant pathways in this population.

Based on the current and new genes identified in glaucoma, it may be possible to develop an algorithm of SNP risk scores to assess the future risk of POAG in patients, which could be clinically useful. However, despite the tremendous progress, the genetic basis of POAG is still not completely understood and further investigations are needed to identify novel genes and pathways contributing to glaucoma that may help define disease-specific targets and facilitate the development of diagnostic and therapeutic strategies. 
Table 2. Possible pathogenesis role of various genes associated with POAG.

\begin{tabular}{|c|c|c|c|}
\hline Gene & Gene Name & Function & Role in Ophthalmic Diseases \\
\hline PLXDC2 & Plexin Domain Containing 2 & May play a role in tumor angiogenesis & $\begin{array}{l}\text { Possible role through inhibition of angiogenesis and possible } \\
\text { involvement in protecting against inflammation }\end{array}$ \\
\hline TMTC2 & $\begin{array}{c}\text { Transmembrane and Tetratricopeptide } \\
\text { Repeat Containing } 2\end{array}$ & $\begin{array}{l}\text { Protein binding calcium ion } \\
\text { homeostasis }\end{array}$ & Unknown \\
\hline $\mathrm{ZP4}$ & Zona Pellucida Glycoprotein 4 & Signal transducer activity & Unknown \\
\hline$S R B D 1$ & S1 RNA Binding Domain 1 & $\begin{array}{l}\text { Nucleic acid binding, RNA binding, } \\
\text { hydrolase activity, acting on ester bonds }\end{array}$ & $\begin{array}{l}\text { Appears to contribute to glaucomatous optic neuropathy as a } \\
\text { non-IOP-related genetic factor; exact mechanism is not known }\end{array}$ \\
\hline ELOVL5 & ELOVL Fatty Acid Elongase 5 & Catalytic activity & $\begin{array}{l}\text { Appears to contribute to glaucomatous optic neuropathy as a } \\
\text { non-IOP-related genetic factor; exact mechanism is not known }\end{array}$ \\
\hline$C A V 1 / C A V 2$ & Caveolin 1/Caveolin 2 & $\begin{array}{l}\text { Receptor binding, structural molecule } \\
\text { activity }\end{array}$ & $\begin{array}{c}\text { Dysfunction of cellular signaling and transport leading to the } \\
\text { damage in tissues }\end{array}$ \\
\hline$C D K N 2 B-A S$ & Cyclin-Dependent Kinase Inhibitor 2B & Protein coding gene, inhibits CDK4 & $\begin{array}{c}\text { Associated with systemic diseases inside and outside the eyes } \\
\text { causing disruption in cell cycle }\end{array}$ \\
\hline TMCO1 & $\begin{array}{l}\text { Transmembrane And Coiled-Coil Domains } \\
1\end{array}$ & Encoding transmembrane protein & Association with cellular malfunction and oxidative stress \\
\hline SIX1 & SIX Homeobox 1 & $\begin{array}{l}\text { Regulation of cell proliferation, } \\
\text { apoptosis and embryonic development. }\end{array}$ & $\begin{array}{l}\text { Associated with developmental malformation of anterior angle, } \\
\qquad \mathrm{TM} \text { and CB }\end{array}$ \\
\hline NCKAP5 & NCK-Associated Protein 5 & Protein coding gene & Unknown \\
\hline$A B C A 1$ & $\begin{array}{l}\text { ATP-Binding Cassette, Sub-Family A } \\
\text { (ABC1), Member } 1\end{array}$ & Cholesterol carrying out of the cell & $\begin{array}{c}\text { Expressed highly in TM network, thought to be involved in } \\
\text { raising IOP }\end{array}$ \\
\hline AFAP1 & Actin Filament Associated Protein 1 & signaling pathways & Possible involvement in aqueous outflow and IOP \\
\hline GMDS & GDP-Mannose 4,6-Dehydratase & Catalytic activity & $\begin{array}{c}\text { GMDS encodes a protein that is required for the first step in de } \\
\text { novo synthesis of fucose. Fucose is required for diverse } \\
\text { biological functions such as growth factor receptor signalling. } \\
\text { Several studies have suggested the effects of growth factors on } \\
\text { development of glaucoma }\end{array}$ \\
\hline$C D C 7$ & Cell Division Cycle 7 & Phosphorylation & Impairment of cellular function in $\mathrm{CB}, \mathrm{TM}$ and $\mathrm{RGC}$ \\
\hline FNDC3B & Fibronectin Type III Domain Containing 3B & Poly(A) RNA binding & Associated with IOP through as yet unknown mechanism \\
\hline
\end{tabular}


Table 2. Cont.

\begin{tabular}{|c|c|c|c|}
\hline Gene & Gene Name & Function & Role in Ophthalmic Diseases \\
\hline GAS7 & Growth Arrest-Specific 7 & $\begin{array}{l}\text { Protein coding gene } \\
\text { sequence-specific DNA binding } \\
\text { transcription factor activity }\end{array}$ & Involved in developmental and functional impairment of RGC \\
\hline$A B O$ & $\begin{array}{c}\text { ABO Blood Group (Transferase A, Alpha } \\
\text { 1-3-N-Acetylgalactosaminyltransferase; } \\
\text { Transferase B, Alpha } \\
\text { 1-3-Galactosyltransferase) }\end{array}$ & Basis of the $\mathrm{ABO}$ blood group system & $\begin{array}{l}\text { Thought to play a role in IOP elevation; } \\
\text { Exact mechanism is not known }\end{array}$ \\
\hline FAR2 & Fatty Acyl CoA Reductase 2 & Catalytic activity & Unknown \\
\hline GGA3 & $\begin{array}{l}\text { Golgi-Associated, Gamma Adaptin Ear } \\
\text { Containing, ARF Binding Protein } 3\end{array}$ & $\begin{array}{c}\text { Protein sorting and trafficking between } \\
\text { the trans-Golgi network (TGN) } \\
\text { and endosomes }\end{array}$ & Unknown \\
\hline PKDREJ & $\begin{array}{l}\text { Polycystin (PKD) Family Receptor For Egg } \\
\text { Jelly }\end{array}$ & May have a central role in fertilization & Elevated IOP through undetermined mechanism \\
\hline ARHGEF12 & $\begin{array}{l}\text { Rho Guanine Nucleotide Exchange Factor } \\
\text { (GEF) } 12\end{array}$ & $\begin{array}{c}\text { May play a role in the regulation of } \\
\text { RhoA GTPase }\end{array}$ & Elevated IOP through undetermined mechanism \\
\hline ATOH7 & Atonal Homolog 7 & $\begin{array}{l}\text { Involved in the differentiation of retinal } \\
\text { ganglion cells }\end{array}$ & Involved in developmental problems of retinal vasculature \\
\hline SALL1 & Spalt-Like Transcription Factor 1 & Organogenesis & $\begin{array}{l}\text { SALL1 is involved in development of calcium homeostasis in } \\
\text { the endoplasmic reticulum }\end{array}$ \\
\hline RFTN1 & Raftlin, Lipid Raft Linker 1 & $\begin{array}{l}\text { Formation and/or maintenance of } \\
\text { lipid rafts. }\end{array}$ & Related to vertical cup-to-disc ratio \\
\hline CARD10 & $\begin{array}{l}\text { Caspase Recruitment Domain Family, } \\
\text { Member } 10\end{array}$ & Protein binding, receptor signaling & Developmental problems of neuronal tissues \\
\hline COL5A1 & Collagen, Type V, Alpha 1 & Fibril formation & $\begin{array}{l}\text { Associated with malformation of connective tissues leading to } \\
\text { problems in cornea and TM }\end{array}$ \\
\hline ZNF469 & Zinc Finger Protein 469 & Transcriptional regulation & Thought to be involved in central corneal thickness \\
\hline AKAP13 & A Kinase (PRKA) Anchor Protein 13 & $\begin{array}{l}\text { Protein binding, cAMP-dependent } \\
\text { protein kinase activity }\end{array}$ & $\begin{array}{l}\text { Involvement in corneal thickness and disruptions in signaling } \\
\text { pathways in CB, TM and RGCs }\end{array}$ \\
\hline
\end{tabular}


Table 2. Cont

\begin{tabular}{|c|c|c|c|}
\hline Gene & Gene Name & Function & Role in Ophthalmic Diseases \\
\hline COL8A2 & Collagen, Type VIII, Alpha 2 & $\begin{array}{l}\text { Protein binding, extracellular matrix } \\
\text { structural constituent }\end{array}$ & $\begin{array}{c}\text { Associated with malformation of connective tissues leading to } \\
\text { problems in cornea and TM }\end{array}$ \\
\hline NTM & Neurotrimin & Protein binding & Unknown \\
\hline$A P O E$ & Apolipoprotein E & Protein binding, receptor binding & $\begin{array}{l}\text { Role in oxidative stress and disrupted cellular homeostasis in } \\
\text { CB, TM, LC and RGC }\end{array}$ \\
\hline CHEK2 & Checkpoint Kinase 2 & Protein kinase activity & $\begin{array}{l}\text { High expression is associated with problems in optic nerve and } \\
\text { cup disk ratio }\end{array}$ \\
\hline FBN1 & Fibrillin 1 & $\begin{array}{l}\text { Extracellular matrix structural } \\
\text { constituent }\end{array}$ & $\begin{array}{l}\text { Mutations in FBN1 could cause backward bowing by } \\
\text { compromising the mechanical properties of the iris }\end{array}$ \\
\hline GSTT1 & Glutathione S-Transferase Theta 1 & Glutathione transferase activity & Oxidative stress in all the POAG-involved tissues \\
\hline NTF4 & Neurotrophin 4 & Protein binding, receptor binding & Retinal ganglion cells survival and apoptosis \\
\hline OPA1 & Optic Atrophy 1 & Protein binding & Involved in Oxidative stress in cornea, $\mathrm{CB}$ and $\mathrm{TM}$ \\
\hline PAX6 & Paired Box 6 & $\begin{array}{l}\text { Sequence-specific DNA binding RNA } \\
\text { polymerase II transcription factor } \\
\text { activity }\end{array}$ & Developmental impairment of neuro ophthalmic system \\
\hline PLXDC2 & Plexin Domain Containing 2 & Receptor binding & Developmental problems leading to fewer retinal ganglion cells \\
\hline SIX6 & SIX Homeobox 6 & DNA binding, protein binding & $\begin{array}{l}\text { Associated with developmental malformation of anterior angle, } \\
\qquad \mathrm{TM} \text { and CB }\end{array}$ \\
\hline TLR4 & Toll-Like Receptor 4 & Receptor binding & Involved in Oxidative stress and decreased cellular viability \\
\hline TMTC2 & $\begin{array}{c}\text { Transmembrane And Tetratricopeptide } \\
\text { Repeat Containing } 2\end{array}$ & Identical protein binding & $\begin{array}{l}\text { TMTC2 is implicated in calcium homeostasis in the } \\
\text { endoplasmic reticulum }\end{array}$ \\
\hline$T N F \alpha$ & Tumor Necrosis Factor & Protease binding, cytokine activity & $\begin{array}{l}\text { May be activated in reaction to POAG-related indices } \\
\text { (increased IOP, oxidative stress and increase in } \\
\text { disregulation of cellular homeostasis }\end{array}$ \\
\hline VAV2 & Vav 2 Guanine Nucleotide Exchange Factor & $\begin{array}{l}\text { Epidermal growth factor } \\
\text { receptor binding }\end{array}$ & Unknown \\
\hline LOXL1 & Lysyl Oxidase-Like 1 & Copper ion binding & $\begin{array}{l}\text { Through the loss of elastin formation and resulting friction } \\
\text { between the iris and the anterior lens capsule }\end{array}$ \\
\hline ZNF469 & Zinc Finger Protein 469 & DNA binding & $\begin{array}{c}\text { Associated with developmental malformation of connective } \\
\text { tissues leading to problems in cornea and TM }\end{array}$ \\
\hline
\end{tabular}


Table 2. Cont.

\begin{tabular}{|c|c|c|c|}
\hline Gene & Gene Name & Function & Role in Ophthalmic Diseases \\
\hline$Z p 4$ & Zona Pellucida Glycoprotein 4 & Signal transducer activity & Unknown \\
\hline TP53 & Tumor Protein P53 & $\begin{array}{l}\text { Core promoter sequence-specific DNA } \\
\text { binding }\end{array}$ & Unknown \\
\hline NOS3 & Nitric Oxide Synthase 3 (Endothelial Cell) & Receptor binding & $\begin{array}{l}\text { Dysregulation of the vascular tone particularly through } \\
\text { interaction with endothelial nitric oxide synthase and } \\
\text { production of nitric oxide (NO) in the vascular endothelia. } \\
\text { This may lead to decreased AH outflow and increased IOP }\end{array}$ \\
\hline$C A T$ & Catalase & Catalytic activity & $\begin{array}{c}\text { Detoxification of reactive oxygen species-linked to POAG } \\
\text { through oxidative stress }\end{array}$ \\
\hline SOD2 & Superoxide Dismutase 2, Mitochondrial & Oxygen binding, DNA binding & Possible role through oxidative stress mechanism \\
\hline OPTN & Optineurin & Protein binding & $\begin{array}{l}\text { Through oxidative stress/the mitochondrial caspase-dependent } \\
\text { cell death }\end{array}$ \\
\hline TTPA & Tocopherol (Alpha) Transfer Protein & Transporter activity & $\begin{array}{l}\text { Linked to vitamin } C \text { loss and that in turn is linked to POAG } \\
\text { development through yet undiscovered mechanism }\end{array}$ \\
\hline$R B P 1$ & Retinol Binding Protein 1, Cellular & Transporter activity, retinoid binding & Through retinol and oxidative stress mechanism \\
\hline MTHFR & $\begin{array}{l}\text { Methylenetetrahydrofolate Reductase } \\
\qquad(\mathrm{NAD}(\mathrm{P}) \mathrm{H})\end{array}$ & $\begin{array}{l}\text { Methylenetetrahydrofolate reductase } \\
(\mathrm{NAD}(\mathrm{P}) \mathrm{H}) \text { activity }\end{array}$ & $\begin{array}{l}\text { Linked through homocysteine level, } \\
\text { link to POAG is not established }\end{array}$ \\
\hline GPX4 & Glutathione Peroxidase 4 & Glutathione peroxidase activity & $\begin{array}{l}\text { Effect on decreased level of vitamins } E \text { and } C \text {. Lower level of } \\
\text { vitamin } C \text { is linked to glaucoma through unknown mechanism. }\end{array}$ \\
\hline SEC14L2 & SEC14-Like 2 (S. Cerevisiae) & Phospholipid binding & $\begin{array}{c}\text { Effect on decreased level of vitamin C. Lower level of vitamin C } \\
\text { is linked to glaucoma through unknown mechanism }\end{array}$ \\
\hline SLC23A1 & $\begin{array}{c}\text { Solute Carrier Family } 23 \text { (Ascorbic Acid } \\
\text { Transporter), Member } 1\end{array}$ & $\begin{array}{l}\text { Nucleobase transmembrane } \\
\text { transporter activity }\end{array}$ & $\begin{array}{l}\text { Effect on decreased level of vitamin C. Lower level of vitamin C } \\
\text { is linked to glaucoma through unknown mechanism }\end{array}$ \\
\hline PMM2 & Phosphomannomutase 2 & Catalytic activity & $\begin{array}{c}\begin{array}{c}\text { Expressed highly in TM network, thought to be involved in } \\
\text { raising IOP }\end{array} \\
\end{array}$ \\
\hline$S L C 23 A 2$ & $\begin{array}{l}\text { Solute Carrier Family } 23 \text { (Ascorbic Acid } \\
\text { Transporter), Member } 2\end{array}$ & $\begin{array}{l}\text { Nucleobase transmembrane } \\
\text { transporter activity }\end{array}$ & $\begin{array}{l}\text { May be through lowering the plasma level of vitamin C. } \\
\text { Low level of vitamin } C \text { was found in POAG patients carrying } \\
\text { mutation in this gene. Exact link between low vitamin } C \text { level } \\
\text { and POAG is not determined }\end{array}$ \\
\hline
\end{tabular}


Since the advent of GWAS studies, more and more genes and SNPs have been discovered in association of POAG. However, the usefulness (in term of clinical application and developing therapeutic modalities) of those discoveries is still limited. It will take multiple genotype-phenotype studies in various centers and multiethnic groups before establishing the applicability of those SNPs and/or genes to POAG or POAG clinical indices. As for the development of new therapeutic agents, the process will be lengthy and may take several years before effective therapeutic modalities for POAG are available. The whole process from discovering new genetic markers (SNPs) or genes to developing new therapeutic agents may take several steps and many years. Those steps are: (i) Discover those genes and/or SNPs associated with POAG, which is underway thanks to new emerging technologies in molecular genetics such as exome sequencing and GWAS technologies. This may take up to 10 years to complete; (ii) Establish the association of various SNPs and genes with POAG in various ethnicities, larger cohorts, and in multiple centers. This is important as initial discovery studies are conducted on specific ethnicities and in smaller cohorts; (iii) Conduct functional studies in order to understand how those genes and/or SNPs contribute to POAG pathogenesis; (iv) Develop therapeutic agents based on our understanding of the function of the genes associated with POAG. This step is the longest and expected to take at least 10-15 years. This should not hold us back or make us think less of genetic studies as those may prove to be the only way to improve our current understanding of the etiology of glaucoma and facilitate the development of diagnostic and therapeutic strategies.

Author Contributions: Khaled Abu-Amero and Altaf A. Kondkar reviewed the literature and participated equally in writing the manuscript. Kakarla V. Chalam, supervised the whole process, revised the clinical information reported All three authors approved the final version of the manuscript.

Conflicts of Interest: The authors declare no conflict of interest.

\section{References}

1. Quigley, H.A.; Broman, A.T. The number of people with glaucoma worldwide in 2010 and 2020. Br. J. Ophthalmol. 2006, 90, 262-267. [CrossRef] [PubMed]

2. Kwon, Y.H.; Fingert, J.H.; Kuehn, M.H.; Alward, W.L. Primary open-angle glaucoma. N. Engl. J. Med. 2009, 360, 1113-1124. [CrossRef] [PubMed]

3. Kang, J.H.; Loomis, S.J.; Rosner, B.A.; Wiggs, J.L.; Pasquale, L.R. Comparison of risk factor profiles for primary open-angle glaucoma subtypes defined by pattern of visual field loss: A prospective study. Investig. Ophthalmol. Vis. Sci. 2015, 56, 2439-2448. [CrossRef] [PubMed]

4. Caprioli, J.; Coleman, A.L. Intraocular pressure fluctuation a risk factor for visual field progression at low intraocular pressures in the advanced glaucoma intervention study. Ophthalmology 2008, 115, 1123-1129. [CrossRef] [PubMed]

5. Gordon, M.O.; Beiser, J.A.; Brandt, J.D.; Heuer, D.K.; Higginbotham, E.J.; Johnson, C.A.; Keltner, J.L.; Miller, J.P.; Parrish, R.K., 2nd; Wilson, M.R.; et al. The ocular hypertension treatment study: Baseline factors that predict the onset of primary open-angle glaucoma. Arch. Ophthalmol. 2002, 120, 714-720. [CrossRef] [PubMed]

6. Coleman, A.L.; Gordon, M.O.; Beiser, J.A.; Kass, M.A. Baseline risk factors for the development of primary open-angle glaucoma in the ocular hypertension treatment study. Am. J. Ophthalmol. 2004, 138, 684-685. [CrossRef] [PubMed]

7. Leske, M.C.; Wu, S.Y.; Honkanen, R.; Nemesure, B.; Schachat, A.; Hyman, L.; Hennis, A. Nine-year incidence of open-angle glaucoma in the barbados eye studies. Ophthalmology 2007, 114, 1058-1064. [CrossRef] [PubMed]

8. Miglior, S.; Pfeiffer, N.; Torri, V.; Zeyen, T.; Cunha-Vaz, J.; Adamsons, I. Predictive factors for open-angle glaucoma among patients with ocular hypertension in the european glaucoma prevention study. Ophthalmology 2007, 114, 3-9. [PubMed]

9. Friedman, D.S.; Wolfs, R.C.; O'Colmain, B.J.; Klein, B.E.; Taylor, H.R.; West, S.; Leske, M.C.; Mitchell, P.; Congdon, N.; Kempen, J. Prevalence of open-angle glaucoma among adults in the united states. Arch. Ophthalmol. 2004, 122, 532-538. [PubMed] 
10. Klein, B.E.; Klein, R.; Lee, K.E. Heritability of risk factors for primary open-angle glaucoma: The beaver dam eye study. Investig. Ophthalmol. Vis. Sci. 2004, 45, 59-62. [CrossRef]

11. de Voogd, S.; Ikram, M.K.; Wolfs, R.C.; Jansonius, N.M.; Hofman, A.; de Jong, P.T. Incidence of open-angle glaucoma in a general elderly population: The rotterdam study. Ophthalmology 2005, 112, 1487-1493. [CrossRef] [PubMed]

12. Tielsch, J.M.; Katz, J.; Sommer, A.; Quigley, H.A.; Javitt, J.C. Family history and risk of primary open angle glaucoma. The baltimore eye survey. Arch. Ophthalmol. 1994, 112, 69-73. [CrossRef] [PubMed]

13. Wang, X.; Harmon, J.; Zabrieskie, N.; Chen, Y.; Grob, S.; Williams, B.; Lee, C.; Kasuga, D.; Shaw, P.X.; Buehler, J.; et al. Using the utah population database to assess familial risk of primary open angle glaucoma. Vis. Res. 2010, 50, 2391-2395. [CrossRef] [PubMed]

14. Mukesh, B.N.; McCarty, C.A.; Rait, J.L.; Taylor, H.R. Five-year incidence of open-angle glaucoma: The visual impairment project. Ophthalmology 2002, 109, 1047-1051. [CrossRef]

15. Kapetanakis, V.V.; Chan, M.P.; Foster, P.J.; Cook, D.G.; Owen, C.G.; Rudnicka, A.R. Global variations and time trends in the prevalence of primary open angle glaucoma (poag): A systematic review and meta-analysis. Br. J. Ophthalmol. 2015. [CrossRef] [PubMed]

16. He, M.; Foster, P.J.; Ge, J.; Huang, W.; Zheng, Y.; Friedman, D.S.; Lee, P.S.; Khaw, P.T. Prevalence and clinical characteristics of glaucoma in adult chinese: A population-based study in liwan district, guangzhou. Investig. Ophthalmol. Vis. Sci. 2006, 47, 2782-2788. [CrossRef] [PubMed]

17. Vannorsdall, T.D.; Schretlen, D.J.; Andrejczuk, M.; Ledoux, K.; Bosley, L.V.; Weaver, J.R.; Skolasky, R.L.; Gordon, B. Altering automatic verbal processes with transcranial direct current stimulation. Front. Psychiatry 2012, 3, 73. [CrossRef] [PubMed]

18. Janicijevic-Petrovic, M.A.; Sarenac-Vulovic, T.S.; Janicijevic, K.M.; Vulovic, D.A.; Andrijana, P.B.; Vujic, D.I. Evaluation of central corneal thickness in patients with ocular hypertension and primary open-angle glaucoma. Med. Glas. 2014, 11, 115-119.

19. Dueker, D.K.; Singh, K.; Lin, S.C.; Fechtner, R.D.; Minckler, D.S.; Samples, J.R.; Schuman, J.S. Corneal thickness measurement in the management of primary open-angle glaucoma: A report by the american academy of ophthalmology. Ophthalmology 2007, 114, 1779-1787. [CrossRef] [PubMed]

20. Copt, R.P.; Thomas, R.; Mermoud, A. Corneal thickness in ocular hypertension, primary open-angle glaucoma, and normal tension glaucoma. Arch. Ophthalmol. 1999, 117, 14-16. [CrossRef] [PubMed]

21. Johnson, T.V.; Toris, C.B.; Fan, S.; Camras, C.B. Effects of central corneal thickness on the efficacy of topical ocular hypotensive medications. J. Glaucoma 2008, 17, 89-99. [CrossRef] [PubMed]

22. Shen, L.; Melles, R.B.; Metlapally, R.; Barcellos, L.; Schaefer, C.; Risch, N.; Herrinton, L.J.; Wildsoet, C.; Jorgenson, E. The association of refractive error with glaucoma in a multiethnic population. Ophthalmology 2015. [CrossRef] [PubMed]

23. Mitchell, P.; Lee, A.J.; Rochtchina, E.; Wang, J.J. Open-angle glaucoma and systemic hypertension: The blue mountains eye study. J. Glaucoma 2004, 13, 319-326. [CrossRef] [PubMed]

24. Wong, T.Y.; Klein, B.E.; Klein, R.; Knudtson, M.; Lee, K.E. Refractive errors, intraocular pressure, and glaucoma in a white population. Ophthalmology 2003, 110, 211-217. [CrossRef]

25. Xu, L.; Wang, Y.; Wang, S.; Jonas, J.B. High myopia and glaucoma susceptibility the beijing eye study. Ophthalmology 2007, 114, 216-220. [CrossRef] [PubMed]

26. Boland, M.V.; Quigley, H.A. Risk factors and open-angle glaucoma: Classification and application. J. Glaucoma 2007, 16, 406-418. [CrossRef] [PubMed]

27. Klein, B.E.; Klein, R.; Jensen, S.C. Open-angle glaucoma and older-onset diabetes. The beaver dam eye study. Ophthalmology 1994, 101, 1173-1177. [CrossRef]

28. Zhou, M.; Wang, W.; Huang, W.; Zhang, X. Diabetes mellitus as a risk factor for open-angle glaucoma: A systematic review and meta-analysis. PLoS ONE 2014, 9, e102972. [CrossRef] [PubMed]

29. Klein, B.E.; Klein, R.; Knudtson, M.D. Intraocular pressure and systemic blood pressure: Longitudinal perspective: The beaver dam eye study. Br. J. Ophthalmol. 2005, 89, 284-287. [CrossRef] [PubMed]

30. Hayreh, S.S.; Podhajsky, P.; Zimmerman, M.B. Beta-blocker eyedrops and nocturnal arterial hypotension. Am. J. Ophthalmol. 1999, 128, 301-309. [CrossRef]

31. Janssen, S.F.; Gorgels, T.G.; Ramdas, W.D.; Klaver, C.C.; van Duijn, C.M.; Jansonius, N.M.; Bergen, A.A. The vast complexity of primary open angle glaucoma: Disease genes, risks, molecular mechanisms and pathobiology. Prog. Retin Eye Res. 2013, 37, 31-67. [CrossRef] [PubMed] 
32. Takamoto, M.; Araie, M. Genetics of primary open angle glaucoma. Jpn. J. Ophthalmol. 2014, 58, 1-15. [CrossRef] [PubMed]

33. Chang, T.C.; Congdon, N.G.; Wojciechowski, R.; Munoz, B.; Gilbert, D.; Chen, P.; Friedman, D.S.; West, S.K. Determinants and heritability of intraocular pressure and cup-to-disc ratio in a defined older population. Ophthalmology 2005, 112, 1186-1191. [CrossRef] [PubMed]

34. Toh, T.; Liew, S.H.; MacKinnon, J.R.; Hewitt, A.W.; Poulsen, J.L.; Spector, T.D.; Gilbert, C.E.; Craig, J.E.; Hammond, C.J.; Mackey, D.A. Central corneal thickness is highly heritable: The twin eye studies. Investig. Ophthalmol. Vis. Sci. 2005, 46, 3718-3722. [CrossRef] [PubMed]

35. Barkana, Y.; Dorairaj, S. Re: Tham et al.: Global prevalence of glaucoma and projections of glaucoma burden through 2040: A systematic review and meta-analysis (ophthalmology 2014;121:2081-90). Ophthalmology 2015, 122, 40-41. [CrossRef] [PubMed]

36. Tielsch, J.M.; Sommer, A.; Katz, J.; Royall, R.M.; Quigley, H.A.; Javitt, J. Racial variations in the prevalence of primary open-angle glaucoma. The baltimore eye survey. JAMA 1991, 266, 369-374. [CrossRef] [PubMed]

37. Friedman, D.S.; Jampel, H.D.; Munoz, B.; West, S.K. The prevalence of open-angle glaucoma among blacks and whites 73 years and older: The salisbury eye evaluation glaucoma study. Arch. Ophthalmol. 2006, 124, 1625-1630. [CrossRef] [PubMed]

38. Nakano, M.; Ikeda, Y.; Taniguchi, T.; Yagi, T.; Fuwa, M.; Omi, N.; Tokuda, Y.; Tanaka, M.; Yoshii, K.; Kageyama, M.; et al. Three susceptible loci associated with primary open-angle glaucoma identified by genome-wide association study in a japanese population. Proc. Natl. Acad. Sci. USA 2009, 106, 12838-12842. [CrossRef] [PubMed]

39. Meguro, A.; Inoko, H.; Ota, M.; Mizuki, N.; Bahram, S. Genome-wide association study of normal tension glaucoma: Common variants in srbd1 and elovl5 contribute to disease susceptibility. Ophthalmology 2010, 117, 1331-1338. [PubMed]

40. Thorleifsson, G.; Walters, G.B.; Hewitt, A.W.; Masson, G.; Helgason, A.; DeWan, A.; Sigurdsson, A.; Jonasdottir, A.; Gudjonsson, S.A.; Magnusson, K.P.; et al. Common variants near cav1 and cav2 are associated with primary open-angle glaucoma. Nat. Genet. 2010, 42, 906-909. [CrossRef] [PubMed]

41. Burdon, K.P.; Macgregor, S.; Hewitt, A.W.; Sharma, S.; Chidlow, G.; Mills, R.A.; Danoy, P.; Casson, R.; Viswanathan, A.C.; Liu, J.Z.; et al. Genome-wide association study identifies susceptibility loci for open angle glaucoma at tmco1 and cdkn2b-as1. Nat. Genet. 2011, 43, 574-578. [CrossRef] [PubMed]

42. Wiggs, J.L.; Yaspan, B.L.; Hauser, M.A.; Kang, J.H.; Allingham, R.R.; Olson, L.M.; Abdrabou, W.; Fan, B.J.; Wang, D.Y.; Brodeur, W.; et al. Common variants at $9 \mathrm{p} 21$ and $8 \mathrm{q} 22$ are associated with increased susceptibility to optic nerve degeneration in glaucoma. PLoS Genet. 2012, 8, e1002654. [CrossRef] [PubMed]

43. Osman, W.; Low, S.K.; Takahashi, A.; Kubo, M.; Nakamura, Y. A genome-wide association study in the japanese population confirms 9p21 and 14q23 as susceptibility loci for primary open angle glaucoma. Hum. Mol. Genet. 2012, 21, 2836-2842. [CrossRef] [PubMed]

44. Nakano, M.; Ikeda, Y.; Tokuda, Y.; Fuwa, M.; Omi, N.; Ueno, M.; Imai, K.; Adachi, H.; Kageyama, M.; Mori, K.; et al. Common variants in cdkn2b-as1 associated with optic-nerve vulnerability of glaucoma identified by genome-wide association studies in japanese. PLoS ONE 2012, 7, e33389. [CrossRef] [PubMed]

45. Takamoto, M.; Kaburaki, T.; Mabuchi, A.; Araie, M.; Amano, S.; Aihara, M.; Tomidokoro, A.; Iwase, A.; Mabuchi, F.; Kashiwagi, K.; et al. Common variants on chromosome 9p21 are associated with normal tension glaucoma. PLoS ONE 2012, 7, e40107. [CrossRef] [PubMed]

46. Chen, Y.; Lin, Y.; Vithana, E.N.; Jia, L.; Zuo, X.; Wong, T.Y.; Chen, L.J.; Zhu, X.; Tam, P.O.; Gong, B.; et al. Common variants near abca1 and in pmm2 are associated with primary open-angle glaucoma. Nat. Genet. 2014, 46, 1115-1119. [CrossRef] [PubMed]

47. Gharahkhani, P.; Burdon, K.P.; Fogarty, R.; Sharma, S.; Hewitt, A.W.; Martin, S.; Law, M.H.; Cremin, K.; Bailey, J.N.; Loomis, S.J.; et al. Common variants near abca1, afap1 and gmds confer risk of primary open-angle glaucoma. Nat. Genet. 2014, 46, 1120-1125. [CrossRef] [PubMed]

48. Li, Z.; Allingham, R.R.; Nakano, M.; Jia, L.; Chen, Y.; Ikeda, Y.; Mani, B.; Chen, L.J.; Kee, C.; Garway-Heath, D.F.; et al. A common variant near tgfbr3 is associated with primary open angle glaucoma. Hum. Mol. Genet. 2015, 24, 3880-3892. [CrossRef] [PubMed]

49. van Koolwijk, L.M.; Ramdas, W.D.; Ikram, M.K.; Jansonius, N.M.; Pasutto, F.; Hysi, P.G.; Macgregor, S.; Janssen, S.F.; Hewitt, A.W.; Viswanathan, A.C.; et al. Common genetic determinants of intraocular pressure and primary open-angle glaucoma. PLoS Genet. 2012, 8, e1002611. [CrossRef] [PubMed] 
50. Hysi, P.G.; Cheng, C.Y.; Springelkamp, H.; Macgregor, S.; Bailey, J.N.; Wojciechowski, R.; Vitart, V.; Nag, A.; Hewitt, A.W.; Hohn, R.; et al. Genome-wide analysis of multi-ancestry cohorts identifies new loci influencing intraocular pressure and susceptibility to glaucoma. Nat. Genet. 2014, 46, 1126-1130. [CrossRef] [PubMed]

51. Chen, F.; Klein, A.P.; Klein, B.E.; Lee, K.E.; Truitt, B.; Klein, R.; Iyengar, S.K.; Duggal, P. Exome array analysis identifies cav1/cav2 as a susceptibility locus for intraocular pressure. Investig. Ophthalmol. Vis. Sci. 2015, 56, 544-551. [CrossRef] [PubMed]

52. Springelkamp, H.; Iglesias, A.I.; Cuellar-Partida, G.; Amin, N.; Burdon, K.P.; van Leeuwen, E.M.; Gharahkhani, P.; Mishra, A.; van der Lee, S.J.; Hewitt, A.W.; et al. Arhgef12 influences the risk of glaucoma by increasing intraocular pressure. Hum. Mol. Genet. 2015, 24, 2689-2699. [CrossRef] [PubMed]

53. Ramdas, W.D.; van Koolwijk, L.M.; Ikram, M.K.; Jansonius, N.M.; de Jong, P.T.; Bergen, A.A.; Isaacs, A.; Amin, N.; Aulchenko, Y.S.; Wolfs, R.C.; et al. A genome-wide association study of optic disc parameters. PLoS Genet. 2010, 6, e1000978. [CrossRef] [PubMed]

54. Ramdas, W.D.; van Koolwijk, L.M.; Lemij, H.G.; Pasutto, F.; Cree, A.J.; Thorleifsson, G.; Janssen, S.F.; Jacoline, T.B.; Amin, N.; Rivadeneira, F.; et al. Common genetic variants associated with open-angle glaucoma. Hum. Mol. Genet. 2011, 20, 2464-2471. [CrossRef] [PubMed]

55. Macgregor, S.; Hewitt, A.W.; Hysi, P.G.; Ruddle, J.B.; Medland, S.E.; Henders, A.K.; Gordon, S.D.; Andrew, T.; McEvoy, B.; Sanfilippo, P.G.; et al. Genome-wide association identifies atoh7 as a major gene determining human optic disc size. Hum. Mol. Genet. 2010, 19, 2716-2724. [CrossRef] [PubMed]

56. Khor, C.C.; Ramdas, W.D.; Vithana, E.N.; Cornes, B.K.; Sim, X.; Tay, W.T.; Saw, S.M.; Zheng, Y.; Lavanya, R.; $\mathrm{Wu}, \mathrm{R}$; et al. Genome-wide association studies in asians confirm the involvement of atoh7 and tgfbr3, and further identify card10 as a novel locus influencing optic disc area. Hum. Mol. Genet. 2011, 20, 1864-1872. [CrossRef] [PubMed]

57. Iglesias, A.I.; Springelkamp, H.; van der Linde, H.; Severijnen, L.A.; Amin, N.; Oostra, B.; Kockx, C.E.; van den Hout, M.C.; van Ijcken, W.F.; Hofman, A.; et al. Exome sequencing and functional analyses suggest that six6 is a gene involved in an altered proliferation-differentiation balance early in life and optic nerve degeneration at old age. Hum. Mol. Genet. 2014, 23, 1320-1332. [CrossRef] [PubMed]

58. Vitart, V.; Bencic, G.; Hayward, C.; Skunca Herman, J.; Huffman, J.; Campbell, S.; Bucan, K.; Navarro, P.; Gunjaca, G.; Marin, J.; et al. New loci associated with central cornea thickness include col5a1, akap13 and avgr8. Hum. Mol. Genet. 2010, 19, 4304-4311. [CrossRef] [PubMed]

59. Vithana, E.N.; Aung, T.; Khor, C.C.; Cornes, B.K.; Tay, W.T.; Sim, X.; Lavanya, R.; Wu, R.; Zheng, Y.; Hibberd, M.L.; et al. Collagen-related genes influence the glaucoma risk factor, central corneal thickness. Hum. Mol. Genet. 2011, 20, 649-658. [CrossRef] [PubMed]

60. Ulmer, M.; Li, J.; Yaspan, B.L.; Ozel, A.B.; Richards, J.E.; Moroi, S.E.; Hawthorne, F.; Budenz, D.L.; Friedman, D.S.; Gaasterland, D.; et al. Genome-wide analysis of central corneal thickness in primary open-angle glaucoma cases in the neighbor and glaugen consortia. Investig. Ophthalmol. Vis. Sci. 2012, 53, 4468-4474. [CrossRef] [PubMed]

61. Chen, L.J.; Tam, P.O.; Leung, D.Y.; Fan, A.H.; Zhang, M.; Tham, C.C.; Chiang, S.W.; Fan, B.J.; Wang, N.; Pang, C.P. Snp rs1533428 at 2p16.3 as a marker for late-onset primary open-angle glaucoma. Mol. Vis. 2012, 18, 1629-1639. [PubMed]

62. Kim, K.; Heo, D.W.; Kim, S.; Kim, J.S.; Kim, C.S.; Kang, C. Expansive marker analysis replicating the association of glaucoma susceptibility with human chromosome loci 1q43 and 10p12.31. Eur. J. Hum. Genet. 2014, 22, 409-413. [CrossRef] [PubMed]

63. Fan, B.J.; Wang, D.Y.; Fan, D.S.; Tam, P.O.; Lam, D.S.; Tham, C.C.; Lam, C.Y.; Lau, T.C.; Pang, C.P. Snps and interaction analyses of myocilin, optineurin, and apolipoprotein e in primary open angle glaucoma patients. Mol. Vis. 2005, 11, 625-631. [PubMed]

64. Lam, C.Y.; Fan, B.J.; Wang, D.Y.; Tam, P.O.; Yung Tham, C.C.; Leung, D.Y.; Ping Fan, D.S.; Chiu Lam, D.S.; Pang, C.P. Association of apolipoprotein e polymorphisms with normal tension glaucoma in a chinese population. J. Glaucoma 2006, 15, 218-222. [CrossRef] [PubMed]

65. Lake, S.; Liverani, E.; Desai, M.; Casson, R.; James, B.; Clark, A.; Salmon, J.F. Normal tension glaucoma is not associated with the common apolipoprotein e gene polymorphisms. Br. J. Ophthalmol. 2004, 88, 491-493. [CrossRef] [PubMed] 
66. Cao, D.; Jiao, X.; Liu, X.; Hennis, A.; Leske, M.C.; Nemesure, B.; Hejtmancik, J.F. Cdkn2b polymorphism is associated with primary open-angle glaucoma (poag) in the afro-caribbean population of barbados, west indies. PLoS ONE 2012, 7, e39278. [CrossRef] [PubMed]

67. Mabuchi, F.; Sakurada, Y.; Kashiwagi, K.; Yamagata, Z.; Iijima, H.; Tsukahara, S. Association between genetic variants associated with vertical cup-to-disc ratio and phenotypic features of primary open-angle glaucoma. Ophthalmology 2012, 119, 1819-1825. [CrossRef] [PubMed]

68. Chen, J.H.; Wang, D.; Huang, C.; Zheng, Y.; Chen, H.; Pang, C.P.; Zhang, M. Interactive effects of atoh7 and rftn1 in association with adult-onset primary open-angle glaucoma. Investig. Ophthalmol. Vis. Sci. 2012, 53, 779-785. [CrossRef] [PubMed]

69. Fan, B.J.; Wang, D.Y.; Pasquale, L.R.; Haines, J.L.; Wiggs, J.L. Genetic variants associated with optic nerve vertical cup-to-disc ratio are risk factors for primary open angle glaucoma in a us caucasian population. Investig. Ophthalmol. Vis. Sci. 2011, 52, 1788-1792. [CrossRef] [PubMed]

70. Dimasi, D.P.; Burdon, K.P.; Hewitt, A.W.; Fitzgerald, J.; Wang, J.J.; Healey, P.R.; Mitchell, P.; Mackey, D.A.; Craig, J.E. Genetic investigation into the endophenotypic status of central corneal thickness and optic disc parameters in relation to open-angle glaucoma. Am. J. Ophthalmol. 2012, 154, 833-842. [CrossRef] [PubMed]

71. Wiggs, J.L.; Kang, J.H.; Yaspan, B.L.; Mirel, D.B.; Laurie, C.; Crenshaw, A.; Brodeur, W.; Gogarten, S.; Olson, L.M.; Abdrabou, W.; et al. Common variants near cav1 and cav2 are associated with primary open-angle glaucoma in caucasians from the USA. Hum. Mol. Genet. 2011, 20, 4707-4713. [CrossRef] [PubMed]

72. Loomis, S.J.; Kang, J.H.; Weinreb, R.N.; Yaspan, B.L.; Cooke Bailey, J.N.; Gaasterland, D.; Gaasterland, T.; Lee, R.K.; Lichter, P.R.; Budenz, D.L.; et al. Association of cav1/cav2 genomic variants with primary open-angle glaucoma overall and by gender and pattern of visual field loss. Ophthalmology 2014, 121, 508-516. [CrossRef] [PubMed]

73. Kuehn, M.H.; Wang, K.; Roos, B.; Stone, E.M.; Kwon, Y.H.; Alward, W.L.; Mullins, R.F.; Fingert, J.H. Chromosome 7q31 poag locus: Ocular expression of caveolins and lack of association with poag in a us cohort. Mol. Vis. 2011, 17, 430-435. [PubMed]

74. Burdon, K.P.; Crawford, A.; Casson, R.J.; Hewitt, A.W.; Landers, J.; Danoy, P.; Mackey, D.A.; Mitchell, P.; Healey, P.R.; Craig, J.E. Glaucoma risk alleles at cdkn2b-as1 are associated with lower intraocular pressure, normal-tension glaucoma, and advanced glaucoma. Ophthalmology 2012, 119, 1539-1545. [CrossRef] [PubMed]

75. Desronvil, T.; Logan-Wyatt, D.; Abdrabou, W.; Triana, M.; Jones, R.; Taheri, S.; Del Bono, E.; Pasquale, L.R.; Olivier, M.; Haines, J.L.; et al. Distribution of col8a2 and col8a1 gene variants in caucasian primary open angle glaucoma patients with thin central corneal thickness. Mol. Vis. 2010, 16, 2185-2191. [PubMed]

76. Dimasi, D.P.; Burdon, K.P.; Hewitt, A.W.; Savarirayan, R.; Healey, P.R.; Mitchell, P.; Mackey, D.A.; Craig, J.E. Candidate gene study to investigate the genetic determinants of normal variation in central corneal thickness. Mol. Vis. 2010, 16, 562-569. [PubMed]

77. Rocha, A.V.; Talbot, T.; Magalhaes da Silva, T.; Almeida, M.C.; Menezes, C.A.; di Pietro, G.; Rios-Santos, F. Is the gstm1 null polymorphism a risk factor in primary open angle glaucoma? Mol. Vis. 2011, 17, 1679-1686. [PubMed]

78. Juronen, E.; Tasa, G.; Veromann, S.; Parts, L.; Tiidla, A.; Pulges, R.; Panov, A.; Soovere, L.; Koka, K.; Mikelsaar, A.V. Polymorphic glutathione s-transferase $\mathrm{m} 1$ is a risk factor of primary open-angle glaucoma among estonians. Exp. Eye Res. 2000, 71, 447-452. [CrossRef] [PubMed]

79. Jansson, M.; Rada, A.; Tomic, L.; Larsson, L.I.; Wadelius, C. Analysis of the glutathione s-transferase $\mathrm{m} 1$ gene using pyrosequencing and multiplex pcr-no evidence of association to glaucoma. Exp. Eye Res. 2003, 77, 239-243. [CrossRef]

80. Fan, B.J.; Liu, K.; Wang, D.Y.; Tham, C.C.; Tam, P.O.; Lam, D.S.; Pang, C.P. Association of polymorphisms of tumor necrosis factor and tumor protein p53 with primary open-angle glaucoma. Investig. Ophthalmol. Vis. Sci. 2010, 51, 4110-4116. [CrossRef] [PubMed]

81. Liu, Y.; Liu, W.; Crooks, K.; Schmidt, S.; Allingham, R.R.; Hauser, M.A. No evidence of association of heterozygous ntf4 mutations in patients with primary open-angle glaucoma. Am. J. Hum. Genet. 2010, 86, 498-499. [CrossRef] [PubMed]

82. Rao, K.N.; Kaur, I.; Parikh, R.S.; Mandal, A.K.; Chandrasekhar, G.; Thomas, R.; Chakrabarti, S. Variations in $\mathrm{ntf} 4$, vav2, and vav3 genes are not involved with primary open-angle and primary angle-closure glaucomas in an indian population. Investig. Ophthalmol. Vis. Sci. 2010, 51, 4937-4941. [CrossRef] [PubMed] 
83. Vithana, E.N.; Nongpiur, M.E.; Venkataraman, D.; Chan, S.H.; Mavinahalli, J.; Aung, T. Identification of a novel mutation in the ntf4 gene that causes primary open-angle glaucoma in a chinese population. Mol. Vis. 2010, 16, 1640-1645. [PubMed]

84. Chen, L.J.; Ng, T.K.; Fan, A.H.; Leung, D.Y.; Zhang, M.; Wang, N.; Zheng, Y.; Liang, X.Y.; Chiang, S.W.; Tam, P.O.; et al. Evaluation of ntf4 as a causative gene for primary open-angle glaucoma. Mol. Vis. 2012, 18, 1763-1772. [PubMed]

85. Aung, T.; Ocaka, L.; Ebenezer, N.D.; Morris, A.G.; Krawczak, M.; Thiselton, D.L.; Alexander, C.; Votruba, M.; Brice, G.; Child, A.H.; et al. A major marker for normal tension glaucoma: Association with polymorphisms in the opa1 gene. Hum. Genet. 2002, 110, 52-56. [CrossRef] [PubMed]

86. Mabuchi, F.; Tang, S.; Kashiwagi, K.; Yamagata, Z.; Iijima, H.; Tsukahara, S. The opa1 gene polymorphism is associated with normal tension and high tension glaucoma. Am. J. Ophthalmol. 2007, 143, 125-130. [CrossRef] [PubMed]

87. Yao, W.; Jiao, X.; Hejtmancik, J.F.; Leske, M.C.; Hennis, A.; Nemesure, B. Evaluation of the association between opa1 polymorphisms and primary open-angle glaucoma in barbados families. Mol. Vis. 2006, 12, 649-654. [PubMed]

88. Carnes, M.U.; Liu, Y.P.; Allingham, R.R.; Whigham, B.T.; Havens, S.; Garrett, M.E.; Qiao, C.; Katsanis, N.; Wiggs, J.L.; Pasquale, L.R.; et al. Discovery and functional annotation of six6 variants in primary open-angle glaucoma. PLoS Genet. 2014, 10, e1004372. [CrossRef] [PubMed]

89. Mabuchi, F.; Sakurada, Y.; Kashiwagi, K.; Yamagata, Z.; Iijima, H.; Tsukahara, S. Association between srbd1 and elovl5 gene polymorphisms and primary open-angle glaucoma. Investig. Ophthalmol. Vis. Sci. 2011, 52, 4626-4629. [CrossRef] [PubMed]

90. Takano, Y.; Shi, D.; Shimizu, A.; Funayama, T.; Mashima, Y.; Yasuda, N.; Fukuchi, T.; Abe, H.; Ideta, H.; Zheng, X.; et al. Association of toll-like receptor 4 gene polymorphisms in japanese subjects with primary open-angle, normal-tension, and exfoliation glaucoma. Am. J. Ophthalmol. 2012, 154, 825-832. [CrossRef] [PubMed]

91. Shibuya, E.; Meguro, A.; Ota, M.; Kashiwagi, K.; Mabuchi, F.; Iijima, H.; Kawase, K.; Yamamoto, T.; Nakamura, M.; Negi, A.; et al. Association of toll-like receptor 4 gene polymorphisms with normal tension glaucoma. Investig. Ophthalmol. Vis. Sci. 2008, 49, 4453-4457. [CrossRef]

92. Sharma, S.; Burdon, K.P.; Chidlow, G.; Klebe, S.; Crawford, A.; Dimasi, D.P.; Dave, A.; Martin, S.; Javadiyan, S.; Wood, J.P.; et al. Association of genetic variants in the tmco1 gene with clinical parameters related to glaucoma and characterization of the protein in the eye. Investig. Ophthalmol. Vis. Sci. 2012, 53, 4917-4925. [CrossRef] [PubMed]

93. Ozel, A.B.; Moroi, S.E.; Reed, D.M.; Nika, M.; Schmidt, C.M.; Akbari, S.; Scott, K.; Rozsa, F.; Pawar, H.; Musch, D.C.; et al. Genome-wide association study and meta-analysis of intraocular pressure. Hum. Genet. 2014, 133, 41-57. [CrossRef] [PubMed]

94. Wang, C.Y.; Shen, Y.C.; Wei, L.C.; Lin, K.H.; Feng, S.C.; Yang, Y.Y.; Chiu, C.H.; Tsai, H.Y. Polymorphism in the tnf-alpha(-863) locus associated with reduced risk of primary open angle glaucoma. Mol. Vis. 2012, 18, 779-785. [PubMed]

95. Mossbock, G.; Weger, M.; Moray, M.; Renner, W.; Haller-Schober, E.M.; Mattes, D.; Schmut, O.; Wegscheider, B.; El-Shabrawi, Y. Tnf-alpha promoter polymorphisms and primary open-angle glaucoma. Eye 2006, 20, 1040-1043. [CrossRef] [PubMed]

96. Neamatzadeh, H.; Soleimanizad, R.; Zare-Shehneh, M.; Gharibi, S.; Shekari, A.; Rahimzadeh, A.B. Association between p53 codon 72 (arg72pro) polymorphism and primary open-angle glaucoma in iranian patients. Iran Biomed. J. 2015, 19, 51-56. [PubMed]

97. Emam, W.A.; Zidan, H.E.; Abdulhalim, B.E.; Dabour, S.A.; Ghali, M.A.; Kamal, A.T. Endothelial nitric oxide synthase polymorphisms and susceptibility to high-tension primary open-angle glaucoma in an egyptian cohort. Mol. Vis. 2014, 20, 804-811. [PubMed]

98. Abu-Amero, K.K.; Kondkar, A.A.; Mousa, A.; Osman, E.A.; Al-Obeidan, S.A. Analysis of catalase snp rs1001179 in saudi patients with primary open angle glaucoma. Ophthalmic Genet. 2013, 34, $223-228$. [CrossRef] [PubMed] 
99. Abu-Amero, K.K.; Kondkar, A.A.; Mousa, A.; Osman, E.A.; Al-Obeidan, S.A. Association of mn-sod mutation (c.47t > c) with various poag clinical indices. Ophthalmic Genet. 2014, 35, 85-90. [CrossRef] [PubMed]

100. Abu-Amero, K.K.; Kondkar, A.A.; Mousa, A.; Osman, E.A.; Al-Obeidan, S.A. Lack of association of snp rs4236601 near cav1 and cav2 with poag in a saudi cohort. Mol. Vis. 2012, 18, 1960-1965. [PubMed]

101. Abu-Amero, K.K.; Osman, E.A.; Azad, M.T.; Allingham, R.R.; Hauser, M.A.; Al-Obeidan, S.A. Lack of association between loxl1 gene polymorphisms and primary open angle glaucoma in the saudi arabian population. Ophthalmic Genet. 2012, 33, 130-133. [CrossRef] [PubMed]

102. Abu-Amero, K.K.; Morales, J.; Bosley, T.M. Mitochondrial abnormalities in patients with primary open-angle glaucoma. Invest. Ophthalmol. Vis. Sci. 2006, 47, 2533-2541. [CrossRef] [PubMed]

103. Zanon-Moreno, V.; Asensio-Marquez, E.M.; Ciancotti-Oliver, L.; Garcia-Medina, J.J.; Sanz, P.; Ortega-Azorin, C.; Pinazo-Duran, M.D.; Ordovas, J.M.; Corella, D. Effects of polymorphisms in vitamin e-, vitamin c-, and glutathione peroxidase-related genes on serum biomarkers and associations with glaucoma. Mol. Vis. 2013, 19, 231-242. [PubMed]

104. Zanon-Moreno, V.; Ciancotti-Olivares, L.; Asencio, J.; Sanz, P.; Ortega-Azorin, C.; Pinazo-Duran, M.D.; Corella, D. Association between a slc23a2 gene variation, plasma vitamin c levels, and risk of glaucoma in a mediterranean population. Mol. Vis. 2011, 17, 2997-3004. [PubMed]

105. Abu-Amero, K.K.; Morales, J.; Mohamed, G.H.; Osman, M.N.; Bosley, T.M. Glutathione s-transferase $\mathrm{m} 1$ and $\mathrm{t} 1$ polymorphisms in arab glaucoma patients. Mol. Vis. 2008, 14, 425-430. [PubMed]

106. Unal, M.; Guven, M.; Devranoglu, K.; Ozaydin, A.; Batar, B.; Tamcelik, N.; Gorgun, E.E.; Ucar, D.; Sarici, A. Glutathione s transferase $\mathrm{m} 1$ and $\mathrm{t} 1$ genetic polymorphisms are related to the risk of primary open-angle glaucoma: A study in a turkish population. Br. J. Ophthalmol. 2007, 91, 527-530. [CrossRef] [PubMed]

107. Al-Dabbagh, N.M.; Al-Dohayan, N.; Arfin, M.; Tariq, M. Apolipoprotein e polymorphisms and primary glaucoma in saudis. Mol. Vis. 2009, 15, 912-919. [PubMed]

108. Saglar, E.; Yucel, D.; Bozkurt, B.; Ozgul, R.K.; Irkec, M.; Ogus, A. Association of polymorphisms in apoe, p53, and p21 with primary open-angle glaucoma in turkish patients. Mol. Vis. 2009, 15, 1270-1276. [PubMed]

109. Nilforoushan, N.; Aghapour, S.; Raoofian, R.; Saee Rad, S.; Greene, W.K.; Fakhraie, G.; Heidari, M. Lack of association between the $\mathrm{c} 677 \mathrm{t}$ single nucleotide polymorphism of the mthfr gene and glaucoma in iranian patients. Acta Med. Iran 2012, 50, 208-212. [PubMed]

110. Gibson, J.; Griffiths, H.; De Salvo, G.; Cole, M.; Jacob, A.; Macleod, A.; Yang, Y.; Menon, G.; Cree, A.; Ennis, S.; et al. Genome-wide association study of primary open angle glaucoma risk and quantitative traits. Mol. Vis. 2012, 18, 1083-1092. [PubMed]

111. Huang, W.; Wang, W.; Zhou, M.; Zhang, X. Association of single-nucleotide polymorphism rs4236601 near caveolin 1 and 2 with primary open-angle glaucoma: A meta-analysis. Clin. Exp. Ophthalmol. 2014, 42, 515-521. [CrossRef] [PubMed]

112. Hannon, G.J.; Beach, D. P15ink4b is a potential effector of tgf-beta-induced cell cycle arrest. Nature 1994, 371, 257-261. [CrossRef] [PubMed]

113. Fuchshofer, R. The pathogenic role of transforming growth factor-beta2 in glaucomatous damage to the optic nerve head. Exp. Eye Res. 2011, 93, 165-169. [CrossRef] [PubMed]

114. Ng, S.K.; Casson, R.J.; Burdon, K.P.; Craig, J.E. Chromosome 9p21 primary open-angle glaucoma susceptibility locus: A review. Clin. Exp. Ophthalmol. 2014, 42, 25-32. [CrossRef] [PubMed]

115. Gallardo, M.E.; Lopez-Rios, J.; Fernaud-Espinosa, I.; Granadino, B.; Sanz, R.; Ramos, C.; Ayuso, C.; Seller, M.J.; Brunner, H.G.; Bovolenta, P.; et al. Genomic cloning and characterization of the human homeobox gene six6 reveals a cluster of six genes in chromosome 14 and associates six6 hemizygosity with bilateral anophthalmia and pituitary anomalies. Genomics 1999, 61, 82-91. [CrossRef] [PubMed]

116. Lu, Y.; Vitart, V.; Burdon, K.P.; Khor, C.C.; Bykhovskaya, Y.; Mirshahi, A.; Hewitt, A.W.; Koehn, D.; Hysi, P.G.; Ramdas, W.D.; et al. Genome-wide association analyses identify multiple loci associated with central corneal thickness and keratoconus. Nat. Genet. 2013, 45, 155-163. [CrossRef] [PubMed]

117. Iglesias, A.I.; Springelkamp, H.; Ramdas, W.D.; Klaver, C.C.; Willemsen, R.; van Duijn, C.M. Genes, pathways, and animal models in primary open-angle glaucoma. Eye 2015, 29, 1285-1298. [CrossRef] [PubMed] 
118. Huang, W.; Wang, W.; Zhou, M.; Chen, S.; Zhang, X. Association of glutathione s-transferase polymorphisms (gstm1 and gstt1) with primary open-angle glaucoma: An evidence-based meta-analysis. Gene 2013, 526, 80-86. [CrossRef] [PubMed]

119. Lu, Y.; Shi, Y.; Yin, J.; Huang, Z. Are glutathione s-transferase polymorphisms (gstm1, gstt1) associated with primary open angle glaucoma? A meta-analysis. Gene 2013, 527, 311-315. [CrossRef] [PubMed]

120. Yu, Y.; Weng, Y.; Guo, J.; Chen, G.; Yao, K. Association of glutathione s transferases polymorphisms with glaucoma: A meta-analysis. PLoS ONE 2013, 8, e54037. [CrossRef] [PubMed]

(C) 2015 by the authors; licensee MDPI, Basel, Switzerland. This article is an open access article distributed under the terms and conditions of the Creative Commons by Attribution (CC-BY) license (http:/ / creativecommons.org/licenses/by/4.0/). 